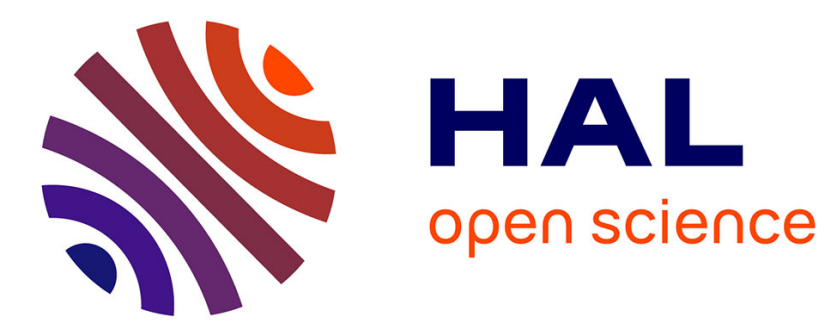

\title{
STOCHASTIC CUCKER-SMALE MODELS: OLD AND NEW
}

Patrick Cattiaux, Fanny Delebecque, Laure Pédèches

\section{To cite this version:}

Patrick Cattiaux, Fanny Delebecque, Laure Pédèches. STOCHASTIC CUCKER-SMALE MODELS: OLD AND NEW. Annals of Applied Probability, 2018, 28 (5), pp.3239-3286. 10.1214/18-AAP1400 . hal-01826717v2

\section{HAL Id: hal-01826717 https://hal.science/hal-01826717v2}

Submitted on 6 Feb 2019

HAL is a multi-disciplinary open access archive for the deposit and dissemination of scientific research documents, whether they are published or not. The documents may come from teaching and research institutions in France or abroad, or from public or private research centers.
L'archive ouverte pluridisciplinaire HAL, est destinée au dépôt et à la diffusion de documents scientifiques de niveau recherche, publiés ou non, émanant des établissements d'enseignement et de recherche français ou étrangers, des laboratoires publics ou privés. 


\title{
STOCHASTIC CUCKER-SMALE MODELS: OLD AND NEW.
}

\section{PATRICK CATTIAUX $\star \quad, \quad$ FANNY DELEBECQUE $\star \quad$, AND LAURE PÉDĖCHES $~$}

\author{
* Université De Toulouse
}

\begin{abstract}
In this paper we revisit and generalize various stochastic models extending the deterministic Cucker-Smale model for self organization. We study flocking and swarming properties. We show how these properties strongly depend on the structure and on the variance of the noise.
\end{abstract}

Key words : Cucker-Smale dynamics, stochastic interacting particles, flocking.

MSC 2010 : 60H10, 82C22, 92C17.

\section{InTRODUCTION, MOTIVATIONS AND EXISTING MODELS.}

In recent years the observation, the description and the modeling of collective motions deserved a lot of attention and consequently produced a huge literature. These kinds of collective behaviors have been observed for several types of populations: humans, fishes, birds, insects, bacteria, macromolecules, cells ... We refer to the beautiful survey [VZ12] for a nice description of various models introduced during the last fifteen years. Despite its fundamental importance, the validation of such models will be ignored in the present work, where we will focus on mathematical properties. However we shall make some small comments on the structure of the models under study throughout the whole paper, and summarize them (with some additional comments) in the final section.

If we read a lot of interesting papers on the subject, it turns out that we do not always completely understand all the mathematical arguments contained in some of them, in particular those dealing with stochastic models. That is why, instead of pointing out these misunderstandings, we decided to make this paper self-contained, at least for the potential readers a little bit familiar with stochastic calculus.

Finally we shall only look at stochastic models where the noise comes from some Brownian motion (or some continuous Ito process). Of course one should also look at jump processes (P.D.M.P. for instance) or fractional processes whose local behavior could introduce other interesting properties.

Let us come to the subject of this work.

The so-called Cucker-Smale model introduced in [CS07a, CS07b] is a mean-field kinetic deterministic model that intends to describe self organization of individuals in a population.

Date: April 21, 2017. 
Originally it is written as

$$
\begin{aligned}
\frac{d}{d t} x_{i}(t) & =v_{i}(t) \\
\frac{d}{d t} v_{i}(t) & =-\frac{\lambda}{N} \sum_{j=1}^{N} \psi_{i j}(t)\left(v_{i}(t)-v_{j}(t)\right) .
\end{aligned}
$$

Here the pair $\left(x_{i}(t), v_{i}(t)\right) \in \mathbb{R}^{d} \otimes \mathbb{R}^{d}$ denotes the pair position/velocity of the "particle" $i \in\{1, \ldots, N\}$ at time $t, \lambda$ is some positive parameter and $\psi_{i j}($.$) is for all (i, j)$ a non-negative function called the communication rate.

In the original model

$$
\psi_{i j}(t)=\psi\left(\left|x_{i}(t)-x_{j}(t)\right|^{2}\right) \quad \text { with } \quad \psi(u)=\frac{1}{(1+u)^{r}} \text { for } r>0 .
$$

The goal was to propose a model for flocking. In the deterministic context, flocking means the following. Introduce the center of mass of the system

$$
\bar{x}(t)=\frac{1}{N} \sum_{j=1}^{N} x_{j}(t) \quad, \quad \bar{v}(t)=\frac{1}{N} \sum_{j=1}^{N} v_{j}(t),
$$

the system (1.1) is said to flock if

$$
\text { for all } i, \quad \lim _{t \rightarrow \infty}\left|v_{i}(t)-\bar{v}(t)\right|=0 \text { and } \sup _{t \geq 0}\left|x_{i}(t)-\bar{x}(t)\right|<+\infty \text {. }
$$

It is known that in the situation of (1.2), flocking occurs for all initial conditions (unconditional flocking) provided $r \leq \frac{1}{2}$, and for some initial conditions otherwise (see [CS07a, CS07b, HL09, HT08]). Of course this is nothing else than convergence to some "equilibrium". Indeed if all initial velocities are the same (hence all equals to $\bar{v}$ ), they do not evolve in time and the motion of the positions block is simply a translation. This is some equilibrium for the model and flocking is thus some kind of convergence to this equilibrium.

A lot of modified models have then been studied in the deterministic context, including delays, no collisions and many other features. Some of them have introduced some randomness in the model, in various ways. The goal of the present paper is to revisit, extend and study these stochastic Cucker-Smale models.

The first question to ask is: where (and why) does randomness enter the game?

The first idea is to consider that each individual has a degree of freedom (or craziness) represented by some random noise independent of the behavior of all other individuals in the population. This leads to the following system for the velocities

$$
d v_{i}(t)=-\frac{\lambda}{N} \sum_{j=1}^{N} \psi_{i j}(t)\left(v_{i}(t)-v_{j}(t)\right) d t+\sigma_{i}(t) d w_{i}(t),
$$

where $\sigma_{i}$ only depends on $\left(x_{i}, v_{i}\right)$ and the $w_{i}$ 's are independent $\mathbb{R}^{d}$ valued noises. This kind of model has been studied in [CM08] for "smooth" noises (actually smooth regularizations of Brownian motions) and in [HLL09] for independent $d$-dimensional standard Brownian motions $w_{i}$ and a constant diffusion matrix $\sigma_{i}$ (actually $\sigma_{i}=\sqrt{D} I d_{d}$ ). The latter case has 
been revisited and completed by one of us in the recent [Péd16]. Here and in what follows the meaning of $d w$ is the Ito differential (we shall come back later to this).

The second idea is to consider that the dynamics of the velocities is perturbed by a noisy environment. This yields the following model

$$
d v_{i}(t)=-\frac{\lambda}{N} \sum_{j=1}^{N} \psi_{i j}(t)\left(v_{i}(t)-v_{j}(t)\right) d t+\sigma_{i}(t) d w(t)
$$

where this time the noise $w$ is the same for all particles.

A very peculiar form of this model is studied in [AH10]. The authors consider therein a noise $w=(W, W, \ldots, W)$ i.e. the same Brownian motion in all the directions of $\mathbb{R}^{d}$ and a diagonal diffusion matrix $\sigma_{i}(t)$ whose diagonal entries are given by the vector $\sigma\left(v_{i}(t)\right)$ where

$$
\sigma(v)=D\left(v-v_{e}\right)
$$

for some constant state $v_{e}$, telling us that the "noise intensity" depends (in a simple way) on the localization of the velocity.

Another idea is to consider that the "infinitesimal" communication rate is perturbed by some noise. This leads to the following model

$$
d v_{i}(t)=-\frac{\lambda}{N} \sum_{j=1}^{N}\left(v_{i}(t)-v_{j}(t)\right)\left(\psi_{i j}(t) d t+\sigma_{i j}(t) d w_{i, j}(t)\right),
$$

where the $w_{i j}$ are again one dimensional noises.

This is done in [TLY14] with $w_{i, j}=w$ for all $i, j$ and with a constant $\sigma_{i, j}=\sigma$, i.e. for some new constant $\bar{\sigma}$,

$$
d v_{i}(t)=-\frac{\lambda}{N} \sum_{j=1}^{N} \psi_{i j}(t)\left(v_{i}(t)-v_{j}(t)\right) d t+\bar{\sigma}\left(v_{i}(t)-\bar{v}(t)\right) d w(t) .
$$

Actually the authors replaced the Ito differential by a Stratonovitch differential. This choice is not really natural since it introduces some repulsive modification on the drift due to the Ito-Stratonovitch correction.

In the recent [EHS15] the authors consider instead $N$ independent one dimensional Brownian noises $w_{i}$ and the following system

$$
d v_{i}(t)=-\frac{\lambda}{N} \sum_{j=1}^{N} \psi_{i j}(t)\left(v_{i}(t)-v_{j}(t)\right) d t+\frac{\sigma_{i}}{N} \sum_{j=1}^{N} \psi_{i j}(t)\left(v_{i}(t)-v_{j}(t)\right) d w_{i}(t)
$$

for constant $\sigma_{i}$. Actually these authors also introduce some delay in the coefficients. A similar model to (1.9) is also discussed in [SL15].

One immediately sees an important difference in nature between all these models. In (1.8) or (1.9), the dynamics $v_{i}(t)=v_{i}(0)=\bar{v}(0)$ for all $i$ is still a solution, hence as for the deterministic system we have some "dynamical" equilibrium. Similarly, if we assume $v_{i}(0)=$ $v_{e}$ for all $i, v_{i}(t)=v_{e}$ furnishes again some dynamical equilibrium for (1.6). In the general case of (1.5) such a trivial solution does no more exist. This shows that the asymptotic behavior of these stochastic systems may be (and actually is) very different. 
The second point is to understand what kind of asymptotic flocking is expected. Indeed since the solutions are random processes, one can look at various behaviors: almost sure behavior, moments behavior, distribution behavior. We will thus introduce three different notions of stochastic flocking

Definition 1.10. Let $\left(x_{i}(t), v_{i}(t)\right)_{i=1, \ldots, N}$ be a $\mathbb{R}^{d} \otimes \mathbb{R}^{d}$ valued stochastic process such that $d x_{i}(t)=v_{i}(t) d t$ for all $i=1, \ldots, N$. Denote by $\bar{v}$ and $\bar{x}$ the centers of masses defined in (1.3). We shall say that:

1) The system is almost surely flocking if (1.4) holds almost surely.

2) The system is flocking in $\mathbb{L}^{p, q}(p, q \geq 1)$ if for all $i$,

$$
\mathbb{E}\left(\left|v_{i}(t)-\bar{v}(t)\right|^{p}\right) \rightarrow 0 \quad \text { as } t \rightarrow+\infty
$$

and

$$
\sup _{t \geq 0} \mathbb{E}\left(\left|x_{i}(t)-\bar{x}(t)\right|^{q}\right)<+\infty .
$$

Actually we will only look at the cases $(p, q)=(1,1),(2,1),(2,2)$. When $q=1$ we simply write $\mathbb{L}^{p}$ flocking.

3) The system is weakly flocking with rate $\varepsilon(R)$ if for all $R>0$ and all $i$,

$$
\limsup _{t \rightarrow+\infty} \mathbb{P}\left(\left|v_{i}(t)-\bar{v}(t)\right|>R\right) \leq \varepsilon(R) .
$$

Remark 1.11. Of course quick enough convergence to 0 for the "centered" velocities is enough to ensure boundedness for the "centered" positions.

For instance,

$$
\mathbb{E}\left(\sup _{t \geq 0}\left|x_{i}(t)-\bar{x}(t)\right|\right) \leq \mathbb{E}\left(\left|x_{i}(0)-\bar{x}(0)\right|\right)+\int_{0}^{+\infty} \mathbb{E}\left(\left|v_{i}(s)-\bar{v}(s)\right|\right) d s<+\infty .
$$

Similarly if for some function $\eta$,

$$
\int_{0}^{+\infty} \mathbb{E}\left(\left|v_{i}(t)-\bar{v}(t)\right|^{2}\right) \eta(t) d t<+\infty
$$

we have

$$
\begin{aligned}
\mathbb{E}\left(\sup _{t \geq 0}\left|x_{i}(t)-\bar{x}(t)\right|^{2}\right) \leq & 2 \mathbb{E}\left(\left|x_{i}(0)-\bar{x}(0)\right|^{2}\right)+2\left(\int_{0}^{+\infty} \mathbb{E}\left(\left|v_{i}(s)-\bar{v}(s)\right|\right) d s\right)^{2} \\
\leq & 2 \mathbb{E}\left(\left|x_{i}(0)-\bar{x}(0)\right|^{2}\right)+ \\
& \quad+2\left(\int_{0}^{+\infty} \mathbb{E}\left(\left|v_{i}(s)-\bar{v}(s)\right|^{2}\right) \eta(s) d s\right)\left(\int_{0}^{+\infty} \eta^{-1}(s) d s\right)
\end{aligned}
$$

so that if in addition $\int_{0}^{+\infty} \eta^{-1}(s) d s<+\infty, \mathbb{E}\left(\sup _{t \geq 0}\left|x_{i}(t)-\bar{x}(t)\right|^{2}\right)<+\infty$ too.

Results in [AH10] concern almost sure flocking, results in [TLY14] concern $\mathbb{L}^{2}$ flocking and those in [Péd16] concern weak flocking.

Another very weak form of stochastic flocking is sometimes discussed: mean-flocking, i.e.

$$
\lim _{t \rightarrow+\infty}\left|\mathbb{E}\left(v_{i}(t)-\bar{v}(t)\right)\right|=0 \quad \text { and } \quad \sup _{t \geq 0}\left|\mathbb{E}\left(x_{i}(t)-\bar{x}(t)\right)\right|<+\infty .
$$

Actually it is this type of flocking which is studied in [HLL09, EHS15]. We confess that we are not really convinced that this kind of property really describes some "collective" behavior, 
though (1.12) can be seen at a first glance as the immediate generalization of deterministic flocking.

In all cases the same strategy of study is used: first look at the motion of the center of mass $\bar{v}(t)$ (the macroscopic level), then look at the fluctuations $\hat{v}_{i}(t)=v_{i}(t)-\bar{v}(t)$ (the microscopic level). As in all the previous works we shall assume in the whole paper that

$$
\text { for all } i, j, \quad \psi_{i j}=\psi_{j i} \text {. }
$$

Under this assumption,

$$
\sum_{i=1}^{N} \sum_{j=1}^{N} \psi_{i j}(t)\left(v_{i}(t)-v_{j}(t)\right)=0
$$

so that the motion of $\bar{v}(t)$ is only driven by the noise.

In order to understand the difference in nature of all these models we shall first look at the simplest case i.e. with a constant communication rate and a constant diffusion coefficient. This is done in the next section 2. In the following section we introduce the notion of swarming and look at its connection with flocking, as it is the case in the deterministic situation. In the two following sections, we still look at constant communication rates but with more general diffusion coefficients for (1.6) and (1.7). This will be the opportunity to introduce the methods that will be mainly used in the general case. In addition, as we shall see in section 6, many results for a non constant communication rate can be deduced from the ones obtained in the constant case. Up to section 6 what is obtained is "unconditional" flocking, that is, without restriction on the initial condition.

Section 6 studies the case of non constant communication rate for the latter two models (1.6) and (1.7). Actually if the communication rate is bounded from below, one can reduce the study to the one with constant communication rate. It it is not bounded from below, we prove some "conditional" flocking results, that is we extend for the first time the corresponding deterministic results to the stochastic situation. The final section deals with comments and simulations.

In order to keep the paper into a reasonable size, we will not discuss here other models of Cucker-Smale type, introducing a mean field term depending on the positions too, or a local mean field dependence as in [MT11]. This will be the aim of future work(s). However, some aspects are already contained in [Péd16] for the model (1.5).

For the sake of simplicity we will assume throughout the paper that the initial conditions $(v(0), x(0))$ are deterministic. All the results can be extended to random initial conditions such that $v(0)-\bar{v}(0)$ and $x(0)-\bar{x}(0)$ are almost surely bounded. We shall also denote by $|y|$ the euclidean norm of a vector $y \in \mathbb{R}^{m}$ whatever $m$ is.

\section{Constant communication rate. A new visit of the existing models.}

In this section we assume that, for all $t$,

$$
\psi_{i j}(t)=\psi_{j i}(t)=\psi>0
$$


Notice that in this situation, under mild assumptions on the diffusion coefficients (ensuring that the stochastic integral is a true martingale) the expectations $\left(\mathbb{E}\left(v_{i}(t)\right), \mathbb{E}\left(x_{i}(t)\right)\right)$ satisfy (1.1) with a constant communication rate, so that one always has mean-flocking.

First we will revisit (and extend) the known results we recalled in the introduction, hence we assume that

(H1) in (1.5) we consider $\sigma_{i}(t)=\sqrt{D} I d_{d}$,

(H2) in (1.6) as in [AH10] we consider $\sigma_{i}(t)=D\left(v_{i}(t)-v_{e}\right)$, but here we assume that $w$ is a $d$-dimensional process $w=\left(w^{1}, \ldots, w^{d}\right)$ such that each $w^{k}$ is a standard linear Brownian motion (we do not make any assumption on the correlations),

(H3) in (1.8) the same assumption for $w$ is made as in (H2),

(H4) in (1.9) the same assumption is made for each $w_{i}=\left(w_{i}^{1}, \ldots, w_{i}^{d}\right)$ (the $w_{i}$ 's being independent) and in addition $\sigma_{i}=\sigma$ for all $i$.

We will prove the following

Theorem 2.2. Consider the previous models assuming (2.1). Then,

(1) If (H1) is satisfied, the system (1.5) is weakly flocking with a rate $\varepsilon(R)$ given by some $\chi^{2}$ tail.

(2) If (H2) is satisfied, the system (1.6) is always almost surely flocking and $\mathbb{L}^{1}$ flocking, but is $\mathbb{L}^{2}$ flocking if and only if $2 \lambda \psi>D^{2}$ (or $v_{i}(0)=\bar{v}(0)$ for all $\left.i\right)$. In this case it is also $\mathbb{L}^{2,2}$ flocking.

In addition $\bar{v}(t)$ goes almost surely to $v_{e}$ as $t$ goes to infinity and $\bar{x}(t)-t v_{e}$ is almost surely bounded.

(3) If (H3) is satisfied, the system (1.8) is always almost surely flocking and $\mathbb{L}^{1}$ flocking, but is $\mathbb{L}^{2}$ flocking if and only if $2 \lambda \psi>\bar{\sigma}^{2}$ (or $v_{i}(0)=\bar{v}(0)$ for all $\left.i\right)$. In this case it is also $\mathbb{L}^{2,2}$ flocking.

In addition $\bar{v}$ is constant hence $\bar{x}(t)$ is linear in $t$.

(4) Assume that the system (1.9) is not at equilibrium i.e. does not satisfy $v_{i}(0)=\bar{v}(0)$ for all $i$. If (H4) is satisfied for (1.9), we have the following situation: define

$$
\alpha=(1-1 / N)(\sigma \psi)^{2}-2 \lambda \psi,
$$

then

(a) if $\alpha<0$ the system is almost surely and $\mathbb{L}^{2,2}$ flocking. In addition the center of mass $\bar{v}(t)$ converges almost surely and in $\mathbb{L}^{1}$ to some given random variable, while $\bar{x}(t)$ has some asymptotic linear behavior.

(b) If $0 \leq \alpha$ the system is not $\mathbb{L}^{2}$ flocking, moreover when $\alpha>0$, the $\mathbb{L}^{2}$ norm of all the $\hat{v}_{i}(t)$ are going to infinity,

(c) if $\frac{2(\sigma \psi)^{2}}{N}>\alpha \geq 0$ the system is almost surely flocking (but not $\mathbb{L}^{2}$ ). This condition implies $N \leq 2$, which is not really interesting.

Remark 2.3. The previous Theorem clearly shows the importance of defining the type of stochastic flocking one wants to get, since on the same elementary model one can have one flocking property and not another one. It also seems that $\mathbb{L}^{2}$ flocking is more demanding. $\diamond$ 
Proof. In the first three cases one can find an explicit solution for the involved stochastic differential equations using that

$$
\frac{1}{N} \sum_{j=1}^{N}\left(v_{i}-v_{j}\right)=v_{i}-\bar{v} .
$$

Let start with (1.8) assuming (H3). It can be rewritten for all $i=1, \ldots, N$ and all $k=1, \ldots, d$,

$$
d v_{i}^{k}(t)=-\lambda \psi\left(v_{i}^{k}(t)-\bar{v}^{k}(t)\right) d t+\bar{\sigma}\left(v_{i}^{k}(t)-\bar{v}^{k}(t)\right) d w^{k}(t) .
$$

In particular $d \bar{v}^{k}(t)=0$ so that $\bar{v}^{k}(t)=\bar{v}^{k}(0)=v_{e}^{k}$ and (2.4) becomes a particular case of (1.6) with $v_{e}=\bar{v}(0)$. This yields the following explicit solution

$$
v_{i}^{k}(t)=\bar{v}^{k}(0)+\left(v_{i}^{k}(0)-\bar{v}^{k}(0)\right) e^{\bar{\sigma} w_{t}^{k}-\left(\frac{1}{2} \bar{\sigma}^{2}+\lambda \psi\right) t} .
$$

Since

$$
\frac{w_{t}^{k}}{t} \rightarrow 0 \text { almost surely as } t \rightarrow+\infty
$$

there is almost sure convergence to the constant center of mass for the velocities. But if $B$. is a linear standard Brownian motion,

$$
\int_{0}^{+\infty} e^{a B_{t}-b t} d t \text { is almost surely bounded for any } a \in \mathbb{R} \text { and } b>0
$$

thanks to the previous remark on the asymptotic behavior of $B_{t} / t$. Thus we have shown almost sure flocking for the model (2.4). Notice that the center of mass of the positions is here simply given by $\bar{x}(t)=\bar{x}(0)+t \bar{v}(0)$.

In addition, on one hand

$$
\begin{aligned}
\mathbb{E}\left(\left|v_{i}^{k}(t)-\bar{v}_{i}^{k}(t)\right|\right) & =\left|v_{i}^{k}(0)-\bar{v}^{k}(0)\right| \mathbb{E}\left(e^{\bar{\sigma} w_{t}^{k}-\frac{1}{2} \bar{\sigma}^{2} t}\right) e^{-2 \lambda \psi t} \\
& =\left|v_{i}^{k}(0)-\bar{v}^{k}(0)\right| e^{-2 \lambda \psi t}
\end{aligned}
$$

while

$$
\begin{aligned}
\mathbb{E}\left(\left(v_{i}^{k}(t)-\bar{v}_{i}^{k}(t)\right)^{2}\right) & =\left(v_{i}^{k}(0)-\bar{v}^{k}(0)\right)^{2} \mathbb{E}\left(e^{2 \bar{\sigma} w_{t}^{k}-\left(\bar{\sigma}^{2}+2 \lambda \psi\right) t}\right) \\
& =\left(v_{i}^{k}(0)-\bar{v}^{k}(0)\right)^{2} e^{\left(-2 \lambda \psi+\bar{\sigma}^{2}\right) t} \mathbb{E}\left(e^{2 \bar{\sigma} w_{t}^{k}-2 \bar{\sigma}^{2} t}\right) \\
& =\left(v_{i}^{k}(0)-\bar{v}^{k}(0)\right)^{2} e^{\left(-2 \lambda \psi+\bar{\sigma}^{2}\right) t} .
\end{aligned}
$$

Hence if $-2 \lambda \psi+\bar{\sigma}^{2} \geq 0$ there is no $\mathbb{L}^{2}$ flocking. For the positions we may use the Remark (1.11) to get $\mathbb{L}^{1}$ flocking. For $\mathbb{L}^{2,2}$ flocking, assuming $-2 \lambda \psi+\bar{\sigma}^{2}<0$, since

$$
e^{\theta t} \mathbb{E}\left(\left|\hat{v}_{i}^{k}(t)\right|^{2}\right) \rightarrow 0 \quad \text { as } t \rightarrow+\infty
$$

for some $\theta>0$, we also have,

$$
\int_{0}^{+\infty} e^{\theta t / 2} \mathbb{E}\left(\left|\hat{v}_{i}^{k}(t)\right|^{2}\right) d t<+\infty,
$$

so that we are again in the situation of the remark. 
Remark 2.7. This result differs from [TLY14] since almost sure flocking occurs in all cases while small noise is required in [TLY14] (that actually does not really study almost sure flocking). This is only due to the fact that, as we said before, the Ito-Stratonovitch correction introduces some repulsive part in the drift in [TLY14].

(1.6) assuming (H2) is thus a little bit more general if $\bar{v}(0) \neq v_{e}$. In this case

$$
\bar{v}^{k}(t)=v_{e}^{k}+\left(\bar{v}^{k}(0)-v_{e}^{k}\right) e^{D w_{t}^{k}-\frac{D^{2} t}{2}}
$$

converges almost surely to $v_{e}^{k}$, and at the microscopic level $\hat{v}_{i}^{k}(t)=v_{i}^{k}(t)-\bar{v}^{k}(t)$ satisfies

$$
d \hat{v}_{i}^{k}(t)=-\lambda \psi \hat{v}_{i}^{k}(t) d t+D \hat{v}_{i}^{k}(t) d w^{k}(t)
$$

so that

$$
\hat{v}_{i}^{k}(t)=\hat{v}_{i}^{k}(0) e^{D w_{t}^{k}-\left(\frac{1}{2} D^{2}+\lambda \psi\right) t}
$$

and we get almost sure flocking as before. This time the center of mass $\bar{v}(t)$ goes almost surely to $v_{e}$ as $t$ goes to infinity and $\bar{x}(t)-t v_{e}$ is almost surely bounded.

The key point here is that, summing up the equations over $i$, we obtain an autonomous S.D.E. for the motion of $\bar{v}$.

Actually the same occurs under (H1) in (1.5). Thanks to the independence of the $w_{i}$ 's, $\bar{v}$ is simply a Brownian motion with covariance matrix $\frac{D}{N} I d_{d}$. We can then get an explicit solution for the motion of $\hat{v}$ which becomes some degenerate $d N$-dimensional Ornstein-Uhlenbeck process (see [Péd16] section 1),

$$
d \hat{v}_{i}(t)=-\lambda \hat{v}_{i}(t) d t+\sqrt{D}\left(1-\frac{1}{N}\right) d w_{i}(t)-\frac{\sqrt{D}}{N} \sum_{j \neq i} d w_{j}(t)
$$

degenerate means that since $\sum_{i} \hat{v}_{i}=0$ the process is an $\mathrm{O}-\mathrm{U}$ process on this subspace. It is then easy to show that $\hat{v}$ is ergodic with a (degenerate but explicit) gaussian invariant distribution so that it is weakly flocking with a rate $\varepsilon(R)$ corresponding to some $\chi^{2}$ tail. However using a Central Limit Theorem one can see that $\hat{x}(t)$ behaves like $\sqrt{t}$ times a gaussian vector (in distribution) so that the Probability for $\hat{x}(t)$ to belong to some bounded set goes to 0 as $t \rightarrow+\infty$ for all bounded sets, that is, weak flocking really only concerns the velocities. We refer to [Péd16] for the details and the explicit computations.

Finally let us look at (1.9) assuming (H4). We first get

$$
d \bar{v}(t)=\frac{\sigma \psi}{N}\left(\sum_{i=1}^{N} v_{i}(t) d w_{i}(t)-\bar{v}(t) \sum_{i=1}^{N} d w_{i}(t)\right)
$$

and then

$$
d \hat{v}_{i}^{k}(t)=-\lambda \psi \hat{v}_{i}^{k}(t) d t+\sigma \psi\left(1-\frac{1}{N}\right) \hat{v}_{i}^{k}(t) d w_{i}(t)-\frac{\sigma \psi}{N} \sum_{j \neq i} \hat{v}_{j}^{k}(t) d w_{j}(t) .
$$


Of course since the coefficients are global Lipschitz, (2.11) admits a unique strong solution. Thus using Ito's formula we get,

$$
\begin{aligned}
z^{k}(t):=\sum_{i=1}^{N}\left(\hat{v}_{i}^{k}\right)^{2}(t)= & z^{k}(0)-2 \lambda \psi \int_{0}^{t} z^{k}(s) d s+2 \sigma \psi \sum_{i=1}^{N}\left(\int_{0}^{t}\left(\hat{v}_{i}^{k}\right)^{2}(s) d w_{i}(s)\right) \\
& -\frac{2 \sigma \psi}{N} \sum_{i, j=1}^{N}\left(\int_{0}^{t}\left(\hat{v}_{i}^{k} \hat{v}_{j}^{k}\right)(s) d w_{j}(s)\right)+(\sigma \psi)^{2}\left(1-\frac{1}{N}\right)^{2} \int_{0}^{t} z^{k}(s) d s \\
& +\frac{(\sigma \psi)^{2}}{N^{2}} \sum_{i \neq j=1}^{N} \int_{0}^{t}\left(\hat{v}_{j}^{k}\right)^{2}(s) d s \\
= & z^{k}(0)+\left(-2 \lambda \psi+(\sigma \psi)^{2}\left(1-\frac{1}{N}\right)\right) \int_{0}^{t} z^{k}(s) d s \\
& +2 \sigma \psi \sum_{i=1}^{N}\left(\int_{0}^{t}\left(\hat{v}_{i}^{k}\right)^{2}(s) d w_{i}(s)\right)
\end{aligned}
$$

since $\sum_{j} \hat{v}_{j}^{k}(s)=0$.

It follows

$$
u^{k}(t):=\mathbb{E}\left(z^{k}(t)\right)=u^{k}(0)+\left(-2 \lambda \psi+(\sigma \psi)^{2}\left(1-\frac{1}{N}\right)\right) \int_{0}^{t} u^{k}(s) d s .
$$

A rigorous proof of (2.13) is straightforward: it is enough to stop the process at the exit time of open balls of radius $R$ (to be sure that the stochastic integrals are true martingales), to take the expectation and then to use the monotone convergence theorem for letting $R$ go to infinity. (2.13) is exactly solved by

$$
u^{k}(t)=u^{k}(0) e^{\alpha t} \quad \text { where } \quad \alpha=(1-1 / N)(\sigma \psi)^{2}-2 \lambda \psi .
$$

We thus have to distinguish three cases: when $\alpha>0 u^{k}(t)$ grows to infinity and there is no $\mathbb{L}^{2}$ flocking, when $\alpha<0$ we may have $\mathbb{L}^{2}$ flocking, when $\alpha=0$ there is no $\mathbb{L}^{2}$ flocking.

We can be more precise. First we have (with an obvious new notation)

$$
\begin{aligned}
u_{i}^{k}(t) & =u_{i}^{k}(0)+\left(-2 \lambda \psi+(\sigma \psi)^{2}\left(1-\frac{1}{N}\right)^{2}\right) \int_{0}^{t} u_{i}^{k}(s) d s+\frac{(\sigma \psi)^{2}}{N^{2}} \sum_{j \neq i} \int_{0}^{t} u_{j}^{k}(s) d s \\
& =u_{i}^{k}(0)+\left(-2 \lambda \psi+(\sigma \psi)^{2}\left(1-\frac{2}{N}\right)\right) \int_{0}^{t} u_{i}^{k}(s) d s+\frac{(\sigma \psi)^{2}}{N^{2}} \int_{0}^{t} u^{k}(s) d s \\
& =u_{i}^{k}(0)+\left(\alpha-\frac{(\sigma \psi)^{2}}{N}\right) \int_{0}^{t} u_{i}^{k}(s) d s+\frac{(\sigma \psi)^{2} u^{k}(0)}{\alpha N^{2}}\left(e^{\alpha t}-1\right)
\end{aligned}
$$

so that it is easily seen (by contradiction for instance) that when $\alpha>0, u_{i}^{k}$ (.) cannot be bounded by some $C e^{\beta t}$ for $\beta<\alpha$. Hence all the $u_{i}^{k}(t)$ are growing to infinity at an exponential rate.

Of course for $\left.\alpha<0, \mathbb{E}\left(\left(\hat{v}_{i}^{k}\right)^{2}(s)\right)\right)$ decays exponentially fast; hence we get $\mathbb{L}^{2,2}$ flocking as before. 
Notice in this case that

$$
\mathbb{E}\left(\left|\bar{v}^{k}(t)-\bar{v}^{k}(0)\right|^{2}\right)=\frac{(\sigma \psi)^{2}}{N^{2}} \int_{0}^{t} u^{k}(s) d s=\frac{(\sigma \psi)^{2}}{N^{2}} \frac{u^{k}(0)}{|\alpha|}\left(1-e^{\alpha t}\right)
$$

is bounded. According to $(2.10),\left(\bar{v}^{k}(t)-\bar{v}^{k}(0)\right)_{t \geq 0}$ is thus a martingale which is bounded in $\mathbb{L}^{2}$. According to Doob's convergence of martingale theorem we know that there exists a random variable $a^{k}$ such that

$$
\left(\bar{v}^{k}(t)-\bar{v}^{k}(0)\right) \rightarrow a^{k} \quad \text { a.s. as } \quad t \rightarrow+\infty .
$$

Since the convergence also holds in $\mathbb{L}^{1}$ we get in addition that $\bar{x}(t)-\bar{x}(0)-t(\bar{v}(0)+a)$ is bounded in $\mathbb{L}^{1}$.

What can be said about the almost sure behavior ? Using Ito formula we get that for all $t<T_{0}$, where $T_{0}$ is the hitting time of 0 for $z^{k}($.$) (notice that for t \geq T_{0}$ one has $z_{t}^{k}=0$ almost surely),

$$
\ln \left(z_{t}^{k}\right)=\ln \left(z^{k}(0)\right)+\alpha t+2 \sigma \psi \sum_{i=1}^{N} \int_{0}^{t} \frac{\left(\hat{v}_{i}^{k}\right)^{2}(s)}{z_{s}^{k}} d w_{i}(s)-2(\sigma \psi)^{2} \sum_{i=1}^{N} \int_{0}^{t} \frac{\left(\hat{v}_{i}^{k}\right)^{4}(s)}{\left(z_{s}^{k}\right)^{2}} d s .
$$

Since $\sum_{i} \beta_{i}^{4} \leq\left(\sum_{i} \beta_{i}^{2}\right)^{2}$, the martingale term

$$
M^{k}(t)=\sum_{i=1}^{N} \int_{0}^{t} \frac{\left(\hat{v}_{i}^{k}\right)^{2}(s)}{z_{s}^{k}} d w_{i}(s)
$$

whose bracket is given by

$$
\left\langle M^{k}\right\rangle(t)=\int_{0}^{t} \frac{\left(\sum_{i=1}^{N} \hat{v}_{i}^{k}\right)^{4}(s)}{\left(\sum_{i=1}^{N}\left(\hat{v}_{i}^{k}\right)^{2}(s)\right)^{2}} d s \leq t
$$

satisfies the two following properties

(1) $t^{-1 / 2} M_{t}^{k}$ is bounded in $\mathbb{L}^{2}$ for $t \in[1,+\infty[$,

(2) $\quad t^{-1} M_{t}^{k} \rightarrow 0$ almost surely as $t \rightarrow+\infty$.

The second point is the standard law of large numbers for martingales.

Using in addition that $\sum_{i=1}^{N} \beta_{i}^{4} \geq \frac{1}{N}\left(\sum_{i=1}^{N} \beta_{i}^{2}\right)^{2}$, we immediately deduce that $z^{k}(t)$ converges almost surely to 0 , hence that we have almost sure flocking, as soon as $\alpha<\frac{2(\sigma \psi)^{2}}{N}$ and that $z^{k}(t)$ goes to infinity (hence no almost sure flocking) if $\alpha>2(\sigma \psi)^{2}$. But the latter cannot occur due to the value of $\alpha$.

\section{Some general properties.}

In this section we introduce some general properties (holding true for any of the model we are considering) that we will use in the sequel.

We start with some simple algebraic remarks:

$$
\sum_{1 \leq i, j \leq N}\left|v_{i}-v_{j}\right|^{2}=2 \sum_{1 \leq i, j \leq N}\left\langle v_{i}, v_{i}-v_{j}\right\rangle=2 N \sum_{i=1}^{N}\left|v_{i}\right|^{2}-2 N^{2}|\bar{v}|^{2}=2 N \sum_{i=1}^{N}\left|v_{i}-\bar{v}\right|^{2},
$$




$$
\sum_{1 \leq i, j \leq N}\left|v_{i}-v_{j}\right|^{2}=\sum_{1 \leq i, j \leq N}\left|\hat{v}_{i}-\hat{v}_{j}\right|^{2}=2 N \sum_{i=1}^{N}\left|\hat{v}_{i}\right|^{2} .
$$

and similarly, if $\psi_{i j}=\psi_{j i}$,

$$
\sum_{1 \leq i, j \leq N} \psi_{i j}\left\langle v_{i}, v_{i}-v_{j}\right\rangle=\frac{1}{2} \sum_{1 \leq i, j \leq N} \psi_{i j}\left|v_{i}-v_{j}\right|^{2},
$$

and more generally

$$
\sum_{1 \leq i, j \leq N} \psi_{i j}\left\langle u_{i}, v_{i}-v_{j}\right\rangle=\frac{1}{2} \sum_{1 \leq i, j \leq N} \psi_{i j}\left\langle u_{i}-u_{j}, v_{i}-v_{j}\right\rangle .
$$

The final (3.4) will allow us, to control in some cases, flocking by a weaker notion called swarming we will define now.

Definition 3.5. Let $\left(x_{i}(t), v_{i}(t)\right)_{i=1, \ldots, N}$ be a $\mathbb{R}^{d} \otimes \mathbb{R}^{d}$ valued stochastic process such that $d x_{i}(t)=v_{i}(t) d t$ for all $i=1, \ldots, N$. Denote by $\bar{v}$ and $\bar{x}$ the centers of masses defined in (1.3). We shall say that:

1) The system is almost surely (resp. $\mathbb{L}^{p}$ ) weakly swarming if

$$
\text { for all } i, \quad \sup _{t \geq 0}\left|v_{i}(t)-\bar{v}(t)\right|<+\infty \text { almost surely }
$$

respectively

$$
\text { for all } i, \quad \sup _{t \geq 0} \mathbb{E}\left(\left|v_{i}(t)-\bar{v}(t)\right|^{p}\right)<+\infty .
$$

2) The system is almost surely (resp. $\mathbb{L}^{p}$, resp. $\mathbb{L}^{p, q}$ ) strongly swarming if in addition, for all $i$,

$$
\sup _{t \geq 0}\left|x_{i}(t)-\bar{x}(t)\right|<+\infty
$$

almost surely, respectively

$$
\sup _{t \geq 0} \mathbb{E}\left(\left|x_{i}(t)-\bar{x}(t)\right|\right)<+\infty,
$$

respectively

$$
\sup _{t \geq 0} \mathbb{E}\left(\left|x_{i}(t)-\bar{x}(t)\right|^{q}\right)<+\infty .
$$

When $\mathbb{E}\left(\sup _{t \geq 0}\left|x_{i}(t)-\bar{x}(t)\right|^{q}\right)<+\infty$ we shall say that the swarming property is uniform (in time).

In some situations, proving swarming is enough to get flocking. Indeed, assume that

$$
\psi_{i j}(v, x)=\psi\left(\left|x_{i}-x_{j}\right|^{2}\right) \text { and define } \Psi(b)=\int_{0}^{b} \psi(a) d a .
$$

We thus have

$$
\Psi\left(\left|x_{i}(t)-x_{j}(t)\right|^{2}\right)-\Psi\left(\left|x_{i}(0)-x_{j}(0)\right|^{2}\right)=2 \int_{0}^{t} \psi_{i j}(s)\left\langle x_{i}(s)-x_{j}(s), v_{i}(s)-v_{j}(s)\right\rangle d s .
$$


Hence, denoting $x_{i j}=x_{i}-x_{j}$ and $v_{i j}=v_{i}-v_{j}$;

$$
\begin{aligned}
\left\langle x_{i j}(t), v_{i j}(t)\right\rangle= & \left\langle x_{i j}(0), v_{i j}(0)\right\rangle-\frac{\lambda}{N} \int_{0}^{t} \sum_{l=1}^{N} \psi_{i l}(s)\left\langle x_{i j}(s), v_{i l}(s)\right\rangle d s \\
& +\frac{\lambda}{N} \int_{0}^{t} \sum_{l=1}^{N} \psi_{j l}(s)\left\langle x_{i j}(s), v_{j l}(s)\right\rangle d s+\int_{0}^{t}\left|v_{i}(s)-v_{j}(s)\right|^{2} d s+M_{i j}(t)
\end{aligned}
$$

where $M_{i j}($.$) is a local martingale term. Let sum up in i, j$. The following term appears

$$
A=-\sum_{i, j, l} \psi_{i l}\left\langle x_{i}-x_{j}, v_{i}-v_{l}\right\rangle+\sum_{i, j, l} \psi_{j l}\left\langle x_{i}-x_{j}, v_{j}-v_{l}\right\rangle .
$$

Let us calculate $A$, first exchanging the role of $i$ and $j$ in the second term,

$$
\begin{aligned}
A & =-2 \sum_{i, j, l} \psi_{i l}\left\langle x_{i}-x_{j}, v_{i}-v_{l}\right\rangle \\
& =-2 N \sum_{i, l} \psi_{i l}\left\langle x_{i}, v_{i}-v_{l}\right\rangle+2 \sum_{j}\left\langle x_{j}, \sum_{i, l} \psi_{i l}\left(v_{i}-v_{l}\right)\right\rangle \\
& =-2 N \sum_{i, l} \psi_{i l}\left\langle x_{i}, v_{i}-v_{l}\right\rangle=-N \sum_{i, l} \psi_{i l}\left\langle x_{i}-x_{l}, v_{i}-v_{l}\right\rangle
\end{aligned}
$$

thanks to (3.4) and since $\sum_{i, l} \psi_{i l}\left(v_{i}-v_{l}\right)=0$.

As usual using some exhausting sequence of stopping times (if it exists) we may integrate up to these random times, for which we get true martingales, take the expectation and then pass to the limit. So we may assume that we have true martingales if we can check that the brackets of the $M_{i j}$ have finite expectation. We shall come back to this point later.

Hence we sum up over all indices and take the expectation, in order to get

$$
\begin{aligned}
\sum_{i, j=1}^{N} \mathbb{E}\left(\left\langle x_{i j}(t), v_{i j}(t)\right\rangle\right)= & \sum_{i, j=1}^{N} \mathbb{E}\left(\left\langle x_{i j}(0), v_{i j}(0)\right\rangle\right)+\int_{0}^{t} \mathbb{E}\left(\sum_{i, j=1}^{N}\left|v_{i}(s)-v_{j}(s)\right|^{2}\right) d s \\
& +\lambda \int_{0}^{t} \sum_{i, j=1}^{N} \mathbb{E}\left(\psi_{i j}(s)\left\langle x_{i}(s)-x_{j}(s), v_{i}(s)-v_{j}(s)\right\rangle\right) d s
\end{aligned}
$$

and finally

$$
\begin{aligned}
\int_{0}^{t} \mathbb{E}\left(\sum_{i, j=1}^{N}\left|v_{i}(s)-v_{j}(s)\right|^{2}\right) d s= & \sum_{i, j=1}^{N}\left(\mathbb{E}\left(\left\langle x_{i j}(t), v_{i j}(t)\right\rangle-\left\langle x_{i j}(0), v_{i j}(0)\right\rangle\right)\right) \\
& -\frac{\lambda}{2} \sum_{i, j=1}^{N} \mathbb{E}\left(\Psi\left(\left|x_{i}(t)-x_{j}(t)\right|^{2}\right)-\Psi\left(\left|x_{i}(0)-x_{j}(0)\right|^{2}\right)\right) \\
\leq & \left.2 N^{2} \max _{i, j}\left(\sup _{s \geq 0} \mathbb{E}\left(\left|x_{i}(s)-x_{j}(s)\right|^{2}\right)\right)^{\frac{1}{2}}\left(\sup _{s \geq 0} \mathbb{E}\left(\left|v_{i}(s)-v_{j}(s)\right|^{2}\right)\right)^{\frac{1}{2}}\right) \\
& -\frac{\lambda}{2} \sum_{i, j=1}^{N} \mathbb{E}\left(\Psi\left(\left|x_{i}(t)-x_{j}(t)\right|^{2}\right)-\Psi\left(\left|x_{i}(0)-x_{j}(0)\right|^{2}\right)\right) .
\end{aligned}
$$


We shall thus use the following elementary Lemma

Lemma 3.10. Let $h: \mathbb{R} \rightarrow \mathbb{R}^{+}$be a $C^{1}$ function with a bounded derivative. If $\int_{0}^{+\infty} h(s) d s<+\infty$, then $h(t) \rightarrow 0$ as $t \rightarrow+\infty$.

We can thus easily deduce that $\mathbb{L}^{2,2}$ swarming implies $\mathbb{L}^{2,2}$ flocking, as soon as $\Psi$ is at most linear, in order to control the second term in the previous sum. Let us state a general result that will be completed in the situations we are looking at later

Lemma 3.11. Consider any of our models. Assume that (3.8) is fulfilled for some bounded function $\psi$. Assume in addition that,

(1) a unique solution $(v(),. x()$.$) exists and is such that for all i, j,\left\langle v_{i}()-.v_{j}(),. x_{i}()-\right.$. $\left.x_{j}().\right\rangle$ is a $\mathbb{L}^{2}$ semi martingale,

(2) for all $i, j, s \mapsto \mathbb{E}\left(\left|v_{i}(s)-v_{j}(s)\right|^{2}\right)$ is well defined and differentiable with a bounded derivative,

(3) the system is $\mathbb{L}^{2,2}$ strongly swarming.

Then the system is $\mathbb{L}^{2,2}$ flocking.

We shall check the required assumptions for each model.

\section{Relaxing (H2) in (1.6) FOR CONStant COMMUniCation RATES.}

In this section we shall study the model (1.6), still assuming that (2.1) is satisfied, but relaxing the assumption (H2). Namely we will consider the following general model

$$
d v_{i}(t)=-\frac{\lambda \psi}{N} \sum_{j=1}^{N}\left(v_{i}(t)-v_{j}(t)\right) d t+\sigma\left(v_{i}(t), x_{i}(t)\right) d w(t),
$$

where $w$ is a $d$-dimensional Brownian motion (the same for all the particles). That is, we consider that the dynamics of a particle is perturbed by a noisy environment depending on the position and the velocity of this particle.

Once again the dynamics of the center of mass is given by a (at least local) martingale

$$
d \bar{v}(t)=\frac{1}{N}\left(\sum_{i=1}^{N} \sigma\left(v_{i}(t), x_{i}(t)\right)\right) d w(t):=s(v(t), x(t)) d w(t) .
$$

It follows

$$
d \hat{v}_{i}^{k}(t)=-\lambda \psi \hat{v}_{i}^{k}(t) d t+\sum_{l=1}^{d} \theta_{i}^{k, l}(v(t), x(t)) d w^{l}(t)
$$

where

$$
\theta_{i}^{k, l}(v, x)=\sigma^{k, l}\left(v_{i}, x_{i}\right)-s^{k, l}(v, x)=\frac{1}{N} \sum_{j=1}^{N}\left(\sigma^{k, l}\left(v_{i}, x_{i}\right)-\sigma^{k, l}\left(v_{j}, x_{j}\right)\right) .
$$

Of course we will assume enough regularity on $\sigma$ for (4.1) to admit a unique solution. Notice that if

$$
v_{i}(0)=v_{0} \quad \text { and } \quad x_{i}(0)=x_{0} \quad \text { for all } i,
$$


then the unique solution of (4.1) is given by a dynamic equilibrium $v_{i}(t)=\bar{v}(t)$ and $x_{i}(t)=$ $\bar{x}(t)$ for all $i$, where $(\bar{v}, \bar{x})$ solves

$$
\begin{aligned}
d \bar{v}(t) & =\sigma(\bar{v}(t), \bar{x}(t)) d w(t) \\
d \bar{x}(t) & =\bar{v}(t) d t .
\end{aligned}
$$

There is however a difference with the deterministic model (or the model assuming (H2)): this time one has in general to fix the initial positions to get some equilibrium.

As we did in the first section we define

$$
\begin{aligned}
z^{k}(t):=\sum_{i=1}^{N}\left(\hat{v}_{i}^{k}\right)^{2}(t)= & z^{k}(0)-2 \lambda \psi \int_{0}^{t} z^{k}(s) d s+2 \sum_{i=1}^{N} \sum_{l=1}^{d} \int_{0}^{t} \hat{v}_{i}^{k}(s) \theta_{i}^{k, l}(v(s), x(s)) d w^{l}(s) \\
& +\int_{0}^{t}\left(\sum_{i=1}^{N} \sum_{l=1}^{d}\left(\theta_{i}^{k, l}\right)^{2}(v(s), x(s))\right) d s
\end{aligned}
$$

so that

$$
\begin{aligned}
z(t):=\sum_{k=1}^{d} z^{k}(t)= & z(0)-2 \lambda \psi \int_{0}^{t} z(s) d s+2 \sum_{i=1}^{N} \sum_{l=1}^{d} \int_{0}^{t}\left(\sum_{k=1}^{d} \hat{v}_{i}^{k}(s) \theta_{i}^{k, l}(v(s), x(s))\right) d w^{l}(s) \\
& +\int_{0}^{t}\left(\sum_{k=1}^{d} \sum_{i=1}^{N} \sum_{l=1}^{d}\left(\theta_{i}^{k, l}\right)^{2}(v(s), x(s))\right) d s .
\end{aligned}
$$

Hence

$$
u(t):=\mathbb{E}(z(t))=u(0)-2 \lambda \psi \int_{0}^{t} u(s) d s+\int_{0}^{t} U(v(s), x(s)) d s
$$

where

$$
U(v(s), x(s))=\sum_{k=1}^{d} \sum_{i=1}^{N} \sum_{l=1}^{d} \mathbb{E}\left[\left(\theta_{i}^{k, l}\right)^{2}(v(s), x(s))\right] .
$$

Finally, at least formally (and rigorously up to the first time $z($.$) hits 0$ )

$$
\begin{aligned}
\ln z(t)= & \ln z(0)+2 \sum_{i=1}^{N} \sum_{l=1}^{d} \int_{0}^{t}\left(\sum_{k=1}^{d} \frac{\hat{v}_{i}^{k}(s) \theta_{i}^{k, l}(v(s), x(s))}{z(s)}\right) d w^{l}(s)-2 \lambda \psi t \\
& +\int_{0}^{t}\left(\sum_{i=1}^{N} \sum_{l=1}^{d} \sum_{k=1}^{d} \frac{\left(\theta_{i}^{k, l}\right)^{2}(v(s), x(s))}{z(s)}\right) d s \\
& -2 \int_{0}^{t} \sum_{l=1}^{d}\left(\frac{\left[\sum_{i=1}^{N} \sum_{k=1}^{d} \hat{v}_{i}^{k}(s) \theta_{i}^{k, l}(s)\right]^{2}}{(z(s))^{2}}\right) d s .
\end{aligned}
$$




\subsection{A first natural generalization of (H2).}

Introduce the following assumption

(H2-1) $\sigma$ only depends on $v$ and is Lipschitz continuous, i.e. there exists $K$ such that for all $k, l$, all $\left(v, v^{\prime}\right)$,

$$
\left|\sigma^{k, l}(v)-\sigma^{k, l}\left(v^{\prime}\right)\right| \leq K\left|v-v^{\prime}\right|
$$

In this situation we have

$$
\begin{aligned}
\left|\theta_{i}^{k, l}(v, x)\right| & \leq \frac{1}{N} \sum_{j \neq i}\left|\sigma^{k, l}\left(v_{i}\right)-\sigma^{k, l}(\bar{v})+\sigma^{k, l}(\bar{v})-\sigma^{k, l}\left(v_{j}\right)\right| \\
& \leq \frac{N-1}{N}\left|\sigma^{k, l}\left(v_{i}\right)-\sigma^{k, l}(\bar{v})\right|+\frac{1}{N} \sum_{j \neq i}\left|\sigma^{k, l}\left(v_{j}\right)-\sigma^{k, l}(\bar{v})\right| \\
& \leq K\left|\hat{v}_{i}\right|+\frac{K}{N} \sum_{j=1}^{N}\left|\hat{v}_{j}\right| .
\end{aligned}
$$

Hence

$$
\sum_{i=1}^{N}\left|\theta_{i}^{k, l}(v, x)\right|^{2} \leq K^{2}\left(\sum_{i=1}^{N}\left|\hat{v}_{i}\right|^{2}+\frac{3}{N}\left(\sum_{j=1}^{N}\left|\hat{v}_{j}\right|\right)^{2}\right) \leq 4 K^{2} \sum_{i=1}^{N}\left|\hat{v}_{i}\right|^{2}
$$

and finally

$$
\sum_{k=1}^{d} \sum_{i=1}^{N} \sum_{l=1}^{d}\left(\theta_{i}^{k, l}\right)^{2}(v(t), x(t)) \leq 4 d^{2} K^{2} z(t) .
$$

Of course if $\sigma$ is diagonal, we may replace $d^{2}$ by $d$, and if in addition $\sigma^{k, k}$ only depends on $v^{k}$ we may replace $d$ by 1 (as for (H2)).

Similarly, using Cauchy-Schwartz inequality it is easily seen that

$$
\left(\frac{\left[\sum_{i=1}^{N} \sum_{k=1}^{d} \hat{v}_{i}^{k}(t) \theta_{i}^{k, l}(t)\right]^{2}}{(z(t))^{2}}\right)
$$

is uniformly bounded above.

We may thus use the same arguments as for the end of the previous proof of Theorem 2.2, except that we do no more have any better lower bound for

$$
\left(\frac{\left[\sum_{i=1}^{N} \sum_{k=1}^{d} \hat{v}_{i}^{k}(t) \theta_{i}^{k, l}(t)\right]^{2}}{(z(t))^{2}}\right)
$$

than 0 . We have thus obtained

Theorem 4.8. Assume that (H2-1) is satisfied in (4.1). Then if $2 \lambda \psi>4 K^{2} d^{2}$, the system (4.1) is almost surely and $\mathbb{L}^{2,2}$ flocking.

However, contrary to what happens when (H2) is satisfied, the center of mass $\bar{v}(t)$ does not necessarily converge as $t \rightarrow+\infty$. 
Let us look at a very particular case: the case when $\sigma$ is diagonal and $\sigma^{k, k}(v)=\sigma^{k, k}\left(v^{k}\right)$. We can thus rewrite (4.1)

$$
d v_{i}^{k}(t)=-\frac{\lambda \psi}{N} \sum_{j=1}^{N}\left(v_{i}^{k}(t)-v_{j}^{k}(t)\right) d t+\sigma^{k, k}\left(v_{i}^{k}(t)\right) d w^{k}(t),
$$

i.e. we can look at the system independently for each coordinate $k$, or if one prefers, reduce the problem to the case of one dimensional particles i.e. $d=1$. In the sequel we thus suppress the superscript $k$.

The first elementary remark is that, if $v_{i}(0)=v_{j}(0)$ for some pair $i \neq j$, the uniqueness of the solution shows that $v_{i}(t)=v_{j}(t)$ for all $t$. Using the Markov property, the same holds for $t \geq T$ for any stopping time $T$ such that $v_{i}(T)=v_{j}(T)$. Reordering the indices if necessary we may assume that $v_{1}(0) \leq v_{2}(0) \leq \ldots \leq v_{N}(0)$ so that the dynamics preserves the order of the velocities of the particles. The best quantity to look at is thus $D_{1 N}(t)=v_{N}(t)-v_{1}(t)$ instead of $z(t)$, since $D_{1 N} \geq \hat{v}_{i}$ for all $i$. The dynamics of $D_{1 N}$ is given by

$$
d D_{1 N}(t)=-\lambda \psi D_{1 N}(t) d t+\left(\sigma\left(v_{N}(t)\right)-\sigma\left(v_{1}(t)\right)\right) d w(t) .
$$

Since $D_{1 N}$ is non negative we have

$\ln D_{1 N}(t)=\ln D_{1 N}(0)-\lambda \psi t-\int_{0}^{t} \frac{\left(\sigma\left(v_{N}(s)\right)-\sigma\left(v_{1}(s)\right)\right)^{2}}{2 D_{1, N}^{2}(s)} d s+\int_{0}^{t} \frac{\left(\sigma\left(v_{N}(s)\right)-\sigma\left(v_{1}(s)\right)\right)}{D_{1 N}(s)} d w(s)$,

and

$$
\begin{aligned}
D_{1 N}^{2}(t)= & D_{1 N}^{2}(0)-\int_{0}^{t}\left(2 \lambda \psi D_{1 N}^{2}(s)-\left(\sigma\left(v_{N}(s)\right)-\sigma\left(v_{1}(s)\right)\right)^{2}\right) d s \\
& +\int_{0}^{t} 2 D_{1 N}(s)\left(\sigma\left(v_{N}(s)\right)-\sigma\left(v_{1}(s)\right)\right) d w(s) .
\end{aligned}
$$

Using the same arguments as before we thus have

Theorem 4.10. Assume that (H2-1) is satisfied in (4.9). Then the system (4.9) is always almost surely flocking. If in addition $2 \lambda \psi>K^{2}$, then it is also $\mathbb{L}^{2,2}$ flocking.

\subsection{More general environments.}

One may ask about the physical meaning of a random environment acting on the velocities only. It can be the case for some aerodynamical perturbations for instance. But of course, it is more natural (or at least as natural) to add some random perturbation that depends on the position (and possibly the velocity too) of each particle. We shall now discuss briefly this situation.

Assume for instance that all $\sigma^{k, l}$ are bounded, say by $M$. We thus have

$$
\mathbb{E}\left(z_{t}\right):=u(t) \leq u(0)-2 \lambda \psi \int_{0}^{t} u(s) d s+4 M^{2} N d^{2} t
$$

so that

$$
\limsup _{t \rightarrow+\infty} u(t) \leq \frac{2 M^{2} N d^{2}}{\lambda \psi}
$$


In particular $u($.$) is bounded on \mathbb{R}^{+}$. Hence if all the $\sigma$ 's are bounded in (4.1), the system is $\mathbb{L}^{2}$ weakly swarming, while in general it is hard to say anything about strong swarming. Concerning this last point let us look at some particular case, namely

(H2-2) $\sigma(v, x)=\sigma(x)$ is $C^{1}$ with partial derivatives bounded by $K$ (but the $\sigma^{k, l}$ are not necessarily bounded themselves).

As for (4.9) we may look at each coordinate $k$ individually, i.e. consider a system of $N$ 1-dimensional particles governed by

$$
d v_{i}^{k}(t)=-\frac{\lambda \psi}{N} \sum_{j=1}^{N}\left(v_{i}^{k}(t)-v_{j}^{k}(t)\right) d t+\sum_{l=1}^{d} \sigma^{k, l}\left(x_{i}(t)\right) d w^{l}(t) .
$$

Contrary to the situation of Theorem 4.10, in general the order of the velocities $v_{i}^{k}$ is not preserved by the dynamics, and the only trivial equilibrium is given by $v_{i}(t)=\bar{v}(0)$ and $x_{i}(t)=\bar{x}(0)+t \bar{v}(0)$ for all $t$.

We shall nevertheless look at

$$
v_{i, j}^{k}(t)=v_{i}^{k}(t)-v_{j}^{k}(t) \quad \text { and } \quad x_{i, j}(t)=x_{i}(t)-x_{j}(t)
$$

which solves

$$
d v_{i, j}^{k}(t)=-\lambda \psi v_{i, j}^{k}(t) d t+\sum_{l=1}^{d} \sigma_{i, j}^{k, l}(t) d w_{t}^{l}
$$

where $\sigma_{i, j}^{k, l}(t)=\sigma^{k, l}\left(x_{i}(t)\right)-\sigma^{k, l}\left(x_{j}(t)\right)$. We already know that, if $\sigma$ is bounded, the system is $\mathbb{L}^{2}$ weakly swarming. Here we assume that

$$
\sup _{t>0} \mathbb{E}\left(\left|x_{i}(t)-x_{j}(t)\right|^{2}\right) \leq M_{i, j}^{2}<+\infty
$$

Of course when $\sigma$ is bounded (4.14) implies that the system is $\mathbb{L}^{2}$ strongly swarming. We shall first show that it is still the case when (H2-2) is satisfied.

Let us make some computations: first if $T_{R}$ denotes the first time $\left|v_{i, j}\right|($.$) exceeds R$, we have

$$
\begin{aligned}
\mathbb{E}\left(\left(v_{i, j}^{k}\right)^{2}\left(t \wedge T_{R}\right)\right) & =\mathbb{E}\left(\left(v_{i, j}^{k}\right)^{2}(0)\right)-2 \lambda \psi \mathbb{E}\left(\int_{0}^{t \wedge T_{R}}\left(v_{i, j}^{k}\right)^{2}(s) d s\right)+\mathbb{E}\left(\int_{0}^{t \wedge T_{R}} \sum_{l=1}^{d}\left(\sigma_{i, j}^{k, l}\right)^{2}(s) d s\right) \\
& \leq \mathbb{E}\left(\left(v_{i, j}^{k}\right)^{2}(0)\right)+d K^{2} M_{i, j}^{2} t
\end{aligned}
$$

so that $u_{i, j}^{k}(t)=\mathbb{E}\left(\left(v_{i, j}^{k}\right)^{2}(t)\right)$ is well defined and satisfies

$$
\mathbb{E}\left(\left(v_{i, j}^{k}\right)^{2}(t)\right):=u_{i, j}^{k}(t) \leq u_{i, j}^{k}(0)-2 \lambda \psi \int_{0}^{t} u_{i, j}^{k}(s) d s+d K^{2} M_{i, j}^{2} t
$$

and finally

$$
\limsup _{t \rightarrow+\infty} u_{i, j}^{k}(t) \leq \frac{d K^{2} M_{i, j}^{2}}{2 \lambda \psi}
$$

It follows that

$$
\sup _{t \geq 0} \mathbb{E}\left(\left(v_{i, j}^{k}\right)^{2}(t)\right) \leq N_{i, j}^{2}<+\infty .
$$


Using what precedes we also see that $s \mapsto \mathbb{E}\left(\left(v_{i, j}^{k}\right)^{2}(s)\right)$ is differentiable with

$$
\frac{d}{d s} \mathbb{E}\left(\left(v_{i, j}^{k}\right)^{2}(s)\right)=-2 \lambda \psi \mathbb{E}\left(\left(v_{i, j}^{k}\right)^{2}(s)\right)+\mathbb{E}\left(\sum_{l=1}^{d}\left(\sigma_{i, j}^{k, l}\right)^{2}(s)\right)
$$

which is bounded below by $-2 \lambda \psi N_{i, j}^{2}$ and bounded above by $d M_{i, j}^{2} K^{2}$. Hence we may use Lemma 3.11 in order to get

Lemma 4.16. Consider the system (4.1) under the assumption (H2-2). If for all pair $(i, j)$,

$$
\sup _{t \geq 0} \mathbb{E}\left(\left|x_{i}(t)-x_{j}(t)\right|^{2}\right) \leq M<+\infty
$$

then the system (4.1) is $\mathbb{L}^{2,2}$ flocking.

But we can go further. Indeed, in the situation of the previous lemma, we first of all have

$$
\mathbb{E}\left(\left(v_{i, j}^{k}\right)^{2}(0)\right)+\int_{0}^{+\infty} \mathbb{E}\left(\sum_{l=1}^{d}\left(\sigma_{i, j}^{k, l}\right)^{2}(s)\right) d s=2 \lambda \psi \int_{0}^{+\infty} \mathbb{E}\left(\left(v_{i, j}^{k}\right)^{2}(s)\right) d s<+\infty .
$$

On one hand, using lemma 3.10 again (it is easily seen that the assumptions are satisfied) we thus obtain

$$
\lim _{t \rightarrow+\infty} \mathbb{E}\left(\left(\sigma_{i, j}^{k, l}\right)^{2}(t)\right)=\lim _{t \rightarrow+\infty} \mathbb{E}\left(\left(\sigma^{k, l}\left(x_{i}(t)\right)-\sigma^{k, l}\left(x_{j}(t)\right)\right)^{2}\right)=0 .
$$

On the other hand, as before the martingale $m_{i, j}^{k}(t)=\sum_{l=1}^{d} \int_{0}^{t} \sigma_{i, j}^{k, l}(s) d w^{l}(s)$ converges (as $t \rightarrow+\infty)$ almost surely and in $\mathbb{L}^{2}$ to a random variable $m_{i, j}^{k}$ such that

$$
\mathbb{E}\left[m_{i, j}^{k} \mid \mathcal{F}^{k}(t)\right]=m_{i, j}^{k}(t),
$$

$\mathcal{F}^{k}($.$) being the filtration of the Brownian motion w($.$) . Notice that$

$$
m_{i, j}^{k}(t)=\left(\lambda \psi x_{i, j}^{k}(t)+v_{i, j}^{k}(t)\right)-\left(\lambda \psi x_{i, j}^{k}(0)+v_{i, j}^{k}(0)\right) .
$$

We deduce that $x_{i}^{k}(t)-x_{j}^{k}(t)$ converges in Probability as $t \rightarrow+\infty$ to

$$
\frac{1}{\lambda \psi} m_{i, j}^{k}+\left(\left(x_{i}^{k}(0)-x_{j}^{k}(0)\right)+\frac{1}{\lambda \psi}\left(v_{i}^{k}(0)-v_{j}^{k}(0)\right)\right)
$$

(since $v_{i, j}^{k}(t)$ goes to 0 in $\mathbb{L}^{2}$ hence in Probability). In addition

$$
\lim _{t \rightarrow+\infty} \mathbb{E}\left(\left(x_{i, j}^{k}\right)^{2}(t)\right)=\mathbb{E}\left(\left(x_{i, j}^{k}(0)+\frac{1}{\lambda \psi} v_{i, j}^{k}(0)\right)^{2}\right)+\frac{1}{(\lambda \psi)^{2}} \mathbb{E}\left(\left(m_{i, j}^{k}\right)^{2}\right) .
$$

It follows that the above convergence in Probability also holds in $\mathbb{L}^{p}$ for all $p<2$.

Hence

Proposition 4.21. Consider the system (4.1) under the assumption (H2-2). If for all pair $(i, j)$,

$$
\sup _{t \geq 0} \mathbb{E}\left(\left|x_{i}(t)-x_{j}(t)\right|^{2}\right) \leq M<+\infty
$$

then the system (4.1) satisfies the following

(1) it is $\mathbb{L}^{2,2}$ flocking,

(2) there exists some random vector $\hat{x}(\infty)$ such that $\hat{x}(t)$ converges in $\mathbb{L}^{p}(p<2)$ towards $\hat{x}(\infty)$ as $t \rightarrow+\infty$. 
Remark 4.22. Notice that if $\lim _{t \rightarrow+\infty} \mathbb{E}\left(\left(x_{i, j}^{k}\right)^{2}(t)\right)=0$, then $0=\lambda \psi x_{i, j}^{k}(0)+v_{i, j}^{k}(0)$ and $m_{i, j}^{k}=0$.

If $m_{i, j}^{k}=0$, then $m_{i, j}^{k}(t)=0$ for all $t \geq 0$, so that

$$
v_{i, j}^{k}(t)=v_{i, j}^{k}(0) e^{-\lambda \psi t} \quad ; \quad x_{i, j}^{k}(t)=x_{i, j}^{k}(0)+\frac{v_{i, j}^{k}(0)}{\lambda \psi}\left(1-e^{-\lambda \psi t}\right) .
$$

So

$$
0=\lambda \psi x_{i, j}^{k}(0)+v_{i, j}^{k}(0)=\lambda \psi x_{i, j}^{k}(t)+v_{i, j}^{k}(t) .
$$

But, since $m_{i, j}^{k}(t)=0$ for all $t$, we also have for all $l$,

$$
\sigma^{k, l}\left(x_{i}(t)\right)-\sigma^{k, l}\left(x_{j}(t)\right)=0 \quad \text { for all } t \geq 0 .
$$

In particular if $\sigma^{k, .}: \mathbb{R}^{d} \mapsto \mathbb{R}^{d}$ is one to one, we get $x_{i, j}(t)=0$ for all $t$, hence $v_{i, j}(t)=0$ for all $t$.

Let us illustrate the previous remark with a simple example

Example 4.23. Almost affine diffusion coefficient.

Assume that for some $k$,

$$
\sigma^{k}(x)=A x+B
$$

for some constant invertible matrix $A$ and constant vector $B$. Then, if (4.14) is satisfied for all pair $(i, j),(4.18)$ yields

$$
\lim _{t \rightarrow+\infty} \mathbb{E}\left(\left|x_{i}(t)-x_{j}(t)\right|^{2}\right)=\lim _{t \rightarrow+\infty} \mathbb{E}\left(\mid A^{-1}\left(\sigma^{k}\left(x_{i}(t)\right)-\left.\sigma^{k}\left(x_{j}(t)\right)\right|^{2}\right)=0,\right.
$$

for all pair $(i, j)$. According to the previous remark, the system is thus at equilibrium. Hence

Proposition 4.24. In addition to (H2-2), if for some $k, \sigma^{k}(x)=A x+B$ for some constant invertible matrix $A$ and constant vector $B$, the system (4.1) cannot be strongly $\mathbb{L}^{2,2}$ swarming, except if it is at equilibrium (all coordinates are equal).

More generally (almost) the same occurs if one of the $\sigma^{k}(k$-th row of the matrix $\sigma)$, in addition to be one to one, satisfies the following property: for a sequence $(x(n), y(n)), \sigma^{k}(x(n))-$ $\sigma^{k}(y(n)) \rightarrow 0$ implies $x(n)-y(n) \rightarrow 0$.

To see it, recall that (4.18) implies that $\sigma^{k}\left(x_{i}(t)\right)-\sigma^{k}\left(x_{j}(t)\right) \rightarrow 0$ in Probability. Hence up to a subsequence $t_{n}$ we may assume that it converges almost surely, so that $x_{i}\left(t_{n}\right)-x_{j}\left(t_{n}\right) \rightarrow 0$ almost surely. But since $x_{i}(t)-x_{j}(t)$ goes to some $x_{i, j}(\infty)$ as $t \rightarrow+\infty$ in Probability, we deduce that $x_{i, j}(\infty)=0$. Using Lebesgue's bounded convergence theorem we can thus deduce

Proposition 4.25. In addition to (H2-2), assume that for some $k, \sigma^{k}$ is one to one and satisfies (H2-21): for a sequence $(x(n), y(n)), \sigma^{k}(x(n))-\sigma^{k}(y(n)) \rightarrow 0$ implies $x(n)-y(n) \rightarrow$ 0 .

Then if the system (4.1) is uniformly $\mathbb{L}^{2,2}$ swarming (i.e. $\max _{i, j} \sup _{t \geq 0}\left|x_{i}(t)-x_{j}(t)\right|=M \in$ $\mathbb{L}^{2}$ ), the system (4.1) is at equilibrium (all coordinates are equal).

The latter statement can be extended: if for instance $\sigma^{k}(x(n))-\sigma^{k}(y(n)) \rightarrow 0$ only implies $x^{k}(n)-y^{k}(n) \rightarrow 0$, then the conclusion of the proposition is still true provided the previous property is satisfied for all $k$. 
The previous assumptions on $\sigma$ imply in a sense that it cannot be bounded. Indeed for $d=1$, a smooth one to one function from $\mathbb{R}$ to $\mathbb{R}$ which is bounded, admits a limit at infinity and thus cannot satisfy (H2-21). The typical example of smooth bounded (and presumably interesting from a physical point of view) function is the case of periodic functions we shall look at now.

Example 4.26. Periodic diffusion coefficient.

Assume now that $\sigma$ is $T$-periodic. For $x \in \mathbb{R}^{d}$ we denote $\tilde{x}$ the unique vector in $\left[0, T{ }^{d}\right.$ such that $x^{k}-\tilde{x}^{k}$ belongs to $T \mathbb{Z}$ for all $k=1, \ldots, d$. By $T$-periodic we mean that $\sigma(x)=\sigma(\tilde{x})$. We shall introduce a new "one to one" assumption:

(H2-22). The set

$$
N=\left\{\tilde{z}=\tilde{x}-\tilde{y} \text { such that for all }(k, l), \sigma^{k, l}(x)-\sigma^{k, l}(y)\right\} \text { is reduced to }\{0\} .
$$

For instance if $d=2$, the matrix

$$
\sigma\left(x^{1}, x^{2}\right)=\left(\begin{array}{ll}
\sin \left(x^{1}\right) & \cos \left(x^{2}\right) \\
\cos \left(x^{1}\right) & \sin \left(x^{2}\right)
\end{array}\right)
$$

satisfies (H2-22) with $T=2 \pi$. The matrix

$$
\sigma\left(x^{1}, x^{2}\right)=\left(\begin{array}{ll}
\sin \left(x^{1}\right) & \cos \left(x^{1}\right) \\
\cos \left(x^{2}\right) & \sin \left(x^{2}\right)
\end{array}\right)
$$

also does, but this case reduces after an immediate change of Brownian motion, to the case of a constant diffusion coefficient.

If the system is strongly $\mathbb{L}^{2,2}$ swarming, we can as in the previous example, find some sequence $t_{n}$ such that for all $(k, l), \sigma^{k, l}\left(\tilde{x}_{i}\left(t_{n}\right)\right)-\sigma^{k, l}\left(\tilde{x}_{j}\left(t_{n}\right)\right) \rightarrow 0$ almost surely. According to proposition $4.21, x_{i}(t)-x_{j}(t)$ goes to $x_{i, j}(\infty)$ in probability, so that taking a subsequence of $t_{n}$ if necessary (we still denote by $t_{n}$ ), we may assume that the convergence is almost sure. It follows that $\tilde{x}_{i}\left(t_{n}\right)-\tilde{x}_{j}\left(t_{n}\right)$ goes almost surely to $\tilde{x}_{i, j}(\infty)$.

Thanks to compactness, we have that for each $\omega$ for which both previous convergences hold, extracting another subsequence if necessary both $\tilde{x}_{i}\left(t_{n}^{\prime}, \omega\right)$ and $\tilde{x}_{j}\left(t_{n}^{\prime}, \omega\right)$ converge to limits $\tilde{x}_{i}(\infty, \omega)$ and $\tilde{x}_{j}(\infty, \omega)$, for which, using the continuity of $\sigma$, it holds that $\sigma^{k, l}\left(\tilde{x}_{i}(\infty, \omega)\right)-$ $\sigma^{k, l}\left(\tilde{x}_{j}(\infty, \omega)\right)=0$. If $(\mathrm{H} 2-22)$ is satisfied, we deduce that $\tilde{x}_{i}(\infty, \omega)-\tilde{x}_{j}(\infty, \omega)=0$, i.e $\tilde{x}_{i, j}(\infty, \omega)=0$ for almost all $\omega$. It means that $x_{i, j}(\infty)$ is a random variable taking its values in $(T \mathbb{Z})^{d}$.

The key point now is the following: if we add to $x(0)$ any $\mathbb{L}^{2}$ random vector whose coordinates belong to $(T \mathbb{Z})^{d}$, we do not change the dynamics of the $v($.$) . Hence, replacing all$ $x_{i}(0)$ by $x_{i}^{\prime}(0)=x_{i}(0)+x_{1, i}(\infty)$ (for $i>1$ ), we do not change the dynamics of the $v_{i}$, we do not change the strong swarming property, nor the uniform swarming property, and we get in the limit $x_{1, i}^{\prime}(\infty)=0$, hence for all $(i, j), x_{i, j}^{\prime}(\infty)=0$. But now we may use remark (4.22), periodicity and (H2-22) to conclude that all $x_{i, j}^{\prime}($.$) and all v_{i, j}($.$) are equal to 0$ as soon as $\lim _{t \rightarrow+\infty} \mathbb{E}\left(\left(\left(x^{\prime}\right)_{i, j}^{k}(t)\right)^{2}\right)=0$, which is satisfied, thanks to Lebesgue bounded convergence theorem as soon as the system is uniformly $\mathbb{L}^{2,2}$ swarming. Notice that now any random vector $(v, x)=(0, x)$ which $x$ taking values in $(T \mathbb{Z})^{d N}$ is an equilibrium. We thus have

Proposition 4.27. In addition to (H2-2), assume that $\sigma$ is T periodic and satisfies (H2-22). Then if the system (4.1) is uniformly $\mathbb{L}^{2,2}$ swarming (i.e. $\max _{i, j} \sup _{t \geq 0}\left|x_{i}(t)-x_{j}(t)\right|=M \in$ 
$\mathbb{L}^{2}$ ), the system (4.1) is at equilibrium (all velocities are equal and the differences between the positions belong to $\left.(T \mathbb{Z})^{d}\right)$.

Hence in all situations we are able to handle, uniform swarming does not occur, unless the system is at equilibrium, telling us that for a random environment depending on the positions only, it seems difficult to swarm out of equilibrium.

\section{A general Form of (1.7) FOR COnstant COMmunication Rates.}

We have already seen that the particular form (1.8) of (1.7) with constant communication rate is a particular case of (1.6). Also notice that, still for constant communication rate, when $w_{i, j}=w_{i}$ for all $j$, the $w_{i}$ being independent, and $\sigma_{i, j}=\sigma_{i} \psi_{i, j}$, we recognize (1.9). We shall now look at another case, namely

$$
d v_{i}(t)=-\frac{\lambda \psi}{N} \sum_{j=1}^{N}\left(v_{i}(t)-v_{j}(t)\right) d t+\frac{1}{N} \sum_{j=1}^{N} \sigma_{i j}(t)\left(v_{i}(t)-v_{j}(t)\right) d w_{i, j}(t),
$$

where the $w_{i j}$ are $d$-dimensional noises (here $v w$ is the vector such that each coordinate $(v w)^{k}$ is given by $\left.v^{k} w^{k}\right)$. We shall assume that

$$
\sigma_{i j}=\sigma_{j i} \quad, \quad w_{i, j}=w_{j, i} \quad, \quad \text { and }\left(w_{i, j}\right)_{i<j} \text { are independent. }
$$

The meaning of these assumptions seems a little bit more natural that for the (1.9) model: each pair of individuals $(i, j)$ are interacting symmetrically with a constant communication rate which is perturbed by some noise (we may include $\psi$ in the $\sigma_{i j}$ ), all the interaction noises being independent. Since we are speaking of constant communication rate, we shall also assume that the $\sigma_{i j}$ are constant (more general situations will be discussed in the next section).

As we did before we shall look at $v_{i, j}=v_{i}-v_{j}$ which solves

$$
d v_{i, j}(t)=-\lambda \psi v_{i, j}(t) d t+\frac{1}{N} \sum_{l=1}^{N} \sigma_{i l} v_{i, l}(t) d w_{i, l}(t)-\frac{1}{N} \sum_{m=1}^{N} \sigma_{j m} v_{j, m}(t) d w_{j, m}(t) .
$$

As before we can look separately at each coordinate $\left(v^{k}, x^{k}\right)$. For the sake of simplicity, we skip the superscript $k$ in the sequel, or if one prefers we take $d=1$.

Hence if we define $z(t)=\sum_{1 \leq i, j \leq N}\left(v_{i, j}\right)^{2}(t)$ (we skip the $2 N$ in (3.1)), we have (being careful with the indices for which the Brownian motions are independent on one hand or the same on the other hand)

$$
\begin{aligned}
d z(t)= & -2 \lambda \psi z(t) d t+\frac{4}{N} \sum_{i, j, l=1}^{N} \sigma_{i l} v_{i, l}(t) v_{i, j}(t) d w_{i, l}(t) \\
& +\frac{2}{N^{2}}\left(\sum_{i, j, l=1}^{N} \sigma_{i l}^{2} v_{i, l}^{2}(t)+\sum_{i, j=1}^{N} \sigma_{i j}^{2} v_{i, j}^{2}(t)\right) d t \\
= & -2 \lambda \psi z(t) d t+4 \sum_{i, l=1}^{N} \sigma_{i l} v_{i, l}(t) \hat{v}_{i}(t) d w_{i, l}(t)+\frac{2(N+1)}{N^{2}}\left(\sum_{i, j=1}^{N} \sigma_{i j}^{2} v_{i, j}^{2}(t)\right) d t .
\end{aligned}
$$


It follows

$$
u(t):=\mathbb{E}(z(t))=u(0)-2 \lambda \psi \int_{0}^{t} u(s) d s+\frac{2(N+1)}{N^{2}} \int_{0}^{t}\left(\sum_{i, j=1}^{N} \sigma_{i j}^{2} \mathbb{E}\left(v_{i, j}^{2}(s)\right)\right) d s,
$$

from which we deduce

$$
\left(\frac{(N+1)}{N^{2}} \min _{i, j} \sigma_{i j}^{2}-\lambda \psi\right) \int_{0}^{t} u(s) d s \leq \frac{u(t)-u(0)}{2} \leq-\left(\lambda \psi-\frac{(N+1)}{N^{2}} \max _{i, j} \sigma_{i j}^{2}\right) \int_{0}^{t} u(s) d s .
$$

The latter furnishes conditions for $\mathbb{L}^{2,2}$ flocking or non flocking.

For almost sure flocking we may consider as we did before $\ln (z(t))$ which solves

$$
\begin{aligned}
d(\ln (z(t))= & -2 \lambda \psi d t+4 \sum_{i, l=1}^{N} \frac{\left(\sigma_{i l} v_{i, l} \hat{v}_{i}\right)(t)}{z(t)} d w_{i, l}(t) \\
& +\frac{2(N+1)}{N^{2}} \sum_{i, l=1}^{N} \frac{\left(\sigma_{i l}^{2} v_{i, l}^{2}\right)(t)}{z(t)} d t-4 \sum_{i, l=1}^{N} \frac{\left(\sigma_{i l}^{2} v_{i, l}^{4}\right)(t)}{z^{2}(t)} d t .
\end{aligned}
$$

The non constant part of the drift term can be rewritten

$$
A(t)=\frac{2}{z^{2}(t)}\left(\frac{N+1}{N}\left[\sum_{i, l=1}^{N}\left(\sigma_{i l}^{2} v_{i, l}^{2}\right)(t)\right]\left[\frac{1}{N} \sum_{i, l=1}^{N}\left(v_{i, l}^{2}\right)(t)\right]-2 \sum_{i, l=1}^{N}\left(\sigma_{i l}^{2} v_{i, l}^{4}\right)(t)\right)
$$

so that using again $\sum_{i=1}^{N} \beta_{i}^{4} \geq \frac{1}{N}\left(\sum_{i=1}^{N} \beta_{i}^{2}\right)^{2}$ we get

$$
A(t) \leq \frac{2}{N}\left(\frac{N+1}{N} \max _{i, j} \sigma_{i j}^{2}-2 \min _{i, j} \sigma_{i j}^{2}\right) .
$$

Now we may argue as in the previous section. We have thus obtained

Theorem 5.5. Consider the system (5.1), under the assumption (5.2) and with constant $\sigma_{i j}$. Then

(1) If $\lambda \psi>\frac{N+1}{N^{2}} \max _{i, j} \sigma_{i j}^{2}$ the system is $\mathbb{L}^{2,2}$ flocking.

(2) If $\lambda \psi<\frac{N+1}{N^{2}} \min _{i, j} \sigma_{i j}^{2}$ the system is not $\mathbb{L}^{2}$ flocking.

(3) If $\lambda \psi-\frac{1}{N}\left(\frac{N+1}{N} \max _{i, j} \sigma_{i j}^{2}-2 \min _{i, j} \sigma_{i j}^{2}\right)>0$, the system is almost surely flocking. In particular if $\sigma_{i j}=\sigma$ for all pair $(i, j)$, the system is always almost surely flocking.

Notice that the flocking properties are still the same if we consider bounded processes $\sigma_{i j}($. instead of constants. Also note that we could improve the bounds for almost sure flocking by using a more accurate comparison between $\sum \sigma_{i l}^{2} v_{i, l}^{2}$ and $\sum v_{i, l}^{2}$, but the present statement is easier.

Remark 5.6. If we compare with (1.7) in his (1.8) version, the correspondence is $\bar{\sigma}=\frac{\sigma}{N}$. The comparison for flocking is thus between $\lambda \psi$ and $\sigma^{2} / N^{2}$ and not with $\sigma / N$. Of course this is simply the observation that the variance of the noise is of order $1 / N^{2}$ in (1.8) while it is of order $1 / N$ here. 


\section{General Communication Rate.}

Since we are mainly interested in flocking or swarming properties, we shall only consider models for which such properties may hold for constant communication rate. [Péd16] contains informations on (1.5) for which it is possible to show the existence of stationary solutions (using Ito-Nisio theory for stochastic delayed equations) as well as propagation of chaos when $N$ grows to infinity (also see [BCnC11] for this latter point). If we consider models for random environment, we will only look at the case where the environment depends on the velocity only. Hence we will focus on two type of systems.

First, noisy communication rates i.e.

$$
d v_{i}(t)=-\frac{\lambda}{N} \sum_{j=1}^{N} \psi_{i j}(t)\left(v_{i}(t)-v_{j}(t)\right) d t+\frac{1}{N} \sum_{j=1}^{N} \sigma_{i j}(t)\left(v_{i}(t)-v_{j}(t)\right) d w_{i, j}(t)
$$

where the $w_{i j}$ are $d$-dimensional noises (again $v w$ is the vector such that each coordinate $(v w)^{k}$ is given by $\left.v^{k} w^{k}\right), w_{i, j}=w_{j, i}$ and the $\left(w_{i, j}\right)_{i<j}$ are independent Brownian motions. Next, noisy environment

$$
d v_{i}(t)=-\frac{\lambda}{N} \sum_{j=1}^{N} \psi_{i j}(t)\left(v_{i}(t)-v_{j}(t)\right) d t+\sigma\left(v_{i}(t)\right) d w(t),
$$

where $w$ is a $d$-dimensional Brownian motion.

\subsection{Study of (6.2).}

Consider the model given by (6.2). We shall introduce assumptions ensuring first existence and uniqueness.

Proposition 6.3. Assume that

(1) The processes $\psi_{i j}(t)$ can be written $\psi_{i j}(t)=\psi_{i j}(v(t), x(t))$, where all the functions $\psi_{i j}$ are local Lipschitz, non-negative and satisfy $\psi_{i j}=\psi_{j i}$,

(2) $\sigma$ satisfies (H2-1) i.e. is globally K-Lipschitz or $\sigma$ is local Lipschitz and bounded.

Then, for all initial state $(v(0), x(0)) \in \mathbb{L}^{2}$ the system (6.2) admits a unique non-explosive (global) strong solution.

Proof. Existence of a unique local strong solution is immediate thanks to our assumptions. The only thing to prove is that it is global. Actually it is enough to show that $v($.$) does not$ explode and to this end, as usual, it is enough to show that for all $t \geq 0$,

$$
\sup _{R>0} \mathbb{E}\left(\left|v\left(t \wedge T_{R}\right)\right|^{2}\right)<+\infty
$$


where $T_{R}$ denotes the first (stopping) time $|v()$.$| hits the value R$. Defining $V()=.|v(.)|^{2}$ we have, using Ito's formula and (3.3), that for $t \leq T_{R}$,

$$
\begin{aligned}
d V(t)= & -\frac{\lambda}{N} \sum_{1 \leq i, j \leq N} \psi_{i j}(t)\left|v_{i}(t)-v_{j}(t)\right|^{2} d t+\sum_{i=1}^{N} \operatorname{Trace}\left(\sigma\left(v_{i}(t)\right) \sigma^{*}\left(v_{i}(t)\right)\right) d t \\
& +2\left(\sum_{i=1}^{N} v_{i}^{*}(t) \sigma\left(v_{i}(t)\right)\right) d w(t),
\end{aligned}
$$

where $a^{*}$ denotes the transposed of the vector (or the matrix) $a$. When $\sigma$ is $K$-Lipschitz, $\left|\sigma^{k, l}\left(v_{i}\right)\right| \leq K\left|v_{i}\right|+c$ for all $(k, l)$, so that

$$
\mathbb{E}\left(\left|v\left(t \wedge T_{R}\right)\right|^{2}\right) \leq \mathbb{E}\left(|v(0)|^{2}\right)+C(N)\left(\int_{0}^{t} K \mathbb{E}\left(\left|v\left(s \wedge T_{R}\right)\right|^{2}\right) d s+c t\right)
$$

and the result follows using Gronwall's lemma. When $\sigma$ is bounded the result is immediate.

Remark 6.4. It is worth noticing that if $v_{i}(0)=\bar{v}(0)$ for all $i$, the unique solution of $(6.2)$ is given by $v_{i}(t)=\bar{v}(t), x_{i}(t)=x_{i}(0)+\int_{0}^{t} \bar{v}(s) d s$, where $\bar{v}($.$) solves$

$$
d \bar{v}(t)=\sigma(\bar{v}(t)) d w(t)
$$

This is in full generality the only dynamic equilibrium of the system.

We consider again

$$
z(t)=\sum_{i=1}^{N}\left|v_{i}(t)-\bar{v}(t)\right|^{2}=\frac{1}{2 N} \sum_{1 \leq i, j \leq N}\left|v_{i}(t)-v_{j}(t)\right|^{2}
$$

Using this time (3.1), Ito's formula and (3.3), we obtain

$$
\begin{aligned}
d z(t)= & -\frac{\lambda}{N} \sum_{1 \leq i, j \leq N} \psi_{i j}(t)\left|v_{i}(t)-v_{j}(t)\right|^{2} d t+\sum_{i=1}^{N} \operatorname{Trace}\left(\sigma\left(v_{i}(t)\right) \sigma^{*}\left(v_{i}(t)\right)\right) d t \\
& -N \operatorname{Trace}\left(\left(\frac{1}{N} \sum_{i=1}^{N} \sigma\left(v_{i}(t)\right)\right)\left(\frac{1}{N} \sum_{i=1}^{N} \sigma^{*}\left(v_{i}(t)\right)\right)\right) d t \\
& +2\left(\sum_{i=1}^{N} \hat{v}_{i}^{*}(t) \sigma\left(v_{i}(t)\right)\right) d w(t),
\end{aligned}
$$

(recall that $a^{*}$ denotes the transposed of the vector (or the matrix) $a$ ). But since $\sum_{i} \hat{v}_{i}=0$, we may replace $\sigma\left(v_{i}\right)$ by $\sigma\left(v_{i}\right)-\sigma(\bar{v})$ in the martingale term. After simple manipulations, it 
follows

$$
\begin{aligned}
d z(t)= & -\frac{\lambda}{N} \sum_{1 \leq i, j \leq N} \psi_{i j}(t)\left|v_{i}(t)-v_{j}(t)\right|^{2} d t \\
& +2\left(\sum_{i=1}^{N} \hat{v}_{i}^{*}(t)\left(\sigma\left(v_{i}(t)\right)-\sigma(\bar{v}(t))\right)\right) d w(t) \\
& +\frac{1}{2 N} \operatorname{Trace}\left(\sum_{1 \leq i, j \leq N}\left(\sigma\left(v_{i}(t)\right)-\sigma\left(v_{j}(t)\right)\right)\left(\sigma^{*}\left(v_{i}(t)\right)-\sigma^{*}\left(v_{j}(t)\right)\right)\right) d t,
\end{aligned}
$$

and

$$
\begin{aligned}
d(\ln z)(t)= & -\frac{\lambda}{N} \sum_{1 \leq i, j \leq N} \psi_{i j}(t) \frac{\left|v_{i}(t)-v_{j}(t)\right|^{2}}{z(t)} d t \\
& +2\left(\sum_{i=1}^{N} \frac{\hat{v}_{i}^{*}(t)\left(\sigma\left(v_{i}(t)\right)-\sigma(\bar{v}(t))\right)}{z(t)}\right) d w(t) \\
& +\frac{1}{2 N} \operatorname{Trace}\left(\sum_{1 \leq i, j \leq N} \frac{\left(\sigma\left(v_{i}(t)\right)-\sigma\left(v_{j}(t)\right)\right)\left(\sigma^{*}\left(v_{i}(t)\right)-\sigma^{*}\left(v_{j}(t)\right)\right)}{z(t)}\right) d t \\
& -2 \frac{\left|\sum_{i=1}^{N} \hat{v}_{i}^{*}(t)\left(\sigma\left(v_{i}(t)\right)-\sigma(\bar{v}(t))\right)\right|^{2}}{z^{2}(t)} d t
\end{aligned}
$$

Of course, except for the part of the drift involving the $\psi_{i j}$ 's, these expressions are exactly the same as in subsection 4.1 (in a more compact form). Hence we know how to manage each term except this part of the drift. But of course if we define

$$
\psi_{\min }=\inf _{i, j, v, x} \psi_{i, j}(v, x) \quad \text { and } \quad \psi_{\max }=\sup _{i, j, v, x} \psi_{i, j}(v, x),
$$

we may write, on one hand

$$
\begin{aligned}
\mathbb{E}(z(t)) \leq & \mathbb{E}(z(0))-2 \lambda \psi_{\min } \int_{0}^{t} \mathbb{E}(z(s)) d s \\
& +\frac{1}{2 N} \int_{0}^{t} \operatorname{Trace}\left(\sum_{1 \leq i, j \leq N}\left(\sigma\left(v_{i}(s)\right)-\sigma\left(v_{j}(s)\right)\right)\left(\sigma^{*}\left(v_{i}(s)\right)-\sigma^{*}\left(v_{j}(s)\right)\right)\right) d s
\end{aligned}
$$

and on the other hand

$$
\begin{aligned}
\mathbb{E}(z(t)) \geq & \mathbb{E}(z(0))-2 \lambda \psi_{\max } \int_{0}^{t} \mathbb{E}(z(s)) d s \\
& +\frac{1}{2 N} \int_{0}^{t} \operatorname{Trace}\left(\sum_{1 \leq i, j \leq N}\left(\sigma\left(v_{i}(s)\right)-\sigma\left(v_{j}(s)\right)\right)\left(\sigma^{*}\left(v_{i}(s)\right)-\sigma^{*}\left(v_{j}(s)\right)\right)\right) d s,
\end{aligned}
$$

so that we may argue exactly as in subsubsection 4.1 to study $\mathbb{L}^{2}$ flocking or non flocking. Similarly, we can get an upper bound for $\ln (z(t))$ replacing all $\psi_{i j}(t)$ by $\psi_{\text {min }}$, and a lower 
bound if $\sigma$ is diagonal with linear diagonal terms as in (1.6), and argue exactly as in subsubsection 4.1 and theorem 2.2 (2) in order to study almost sure flocking. This yields the following two results

Theorem 6.8. Assume that $\psi_{i j}(t)=\psi_{i j}(v(t), x(t))$ where all the functions $\psi_{i j}$ are local Lipschitz, non-negative and satisfy $\psi_{i j}=\psi_{j i}$, and that $\sigma$ satisfies (H2-1) i.e. is globally $K$-Lipschitz. Define $\psi_{\min }$ and $\psi_{\max }$ as in (6.7). Then :

(1) if $2 \lambda \psi_{\min }>4 K^{2} d^{2}$ the system (6.2) is almost surely and $\mathbb{L}^{2,2}$ flocking. When $\sigma$ is diagonal we may replace $d^{2}$ by $d$, if in addition the diagonal term $\sigma^{k, k}(v)=\sigma^{k, k}\left(v^{k}\right)$ we may replace $d$ by 1 .

(2) If $\sigma$ is diagonal with linear entries, i.e. $\sigma^{k, k}(v)=D\left(v^{k}-v_{e}^{k}\right)$, the system is always almost surely flocking provided $D \neq 0$.

If $2 \lambda \psi_{\max } \leq D^{2}$, the system is not $\mathbb{L}^{2}$ flocking.

For (2) just remark that, when $\sigma$ is diagonal with linear entries, it holds

$$
\begin{aligned}
& \operatorname{Trace}\left(\sum_{1 \leq i, j \leq N} \frac{\left(\sigma\left(v_{i}(t)\right)-\sigma\left(v_{j}(t)\right)\right)\left(\sigma^{*}\left(v_{i}(t)\right)-\sigma^{*}\left(v_{j}(t)\right)\right)}{z(t)}\right)- \\
- & 2 \frac{\left|\sum_{i=1}^{N} \hat{v}_{i}^{*}(t)\left(\sigma\left(v_{i}(t)\right)-\sigma(\bar{v}(t))\right)\right|^{2}}{z^{2}(t)} \leq-D^{2},
\end{aligned}
$$

so that we get almost sure flocking (looking at $\ln (z(t))$ as soon as $D \neq 0$.

For the $\mathbb{L}^{2}$ non-flocking property it is enough to look at the lower bound for $\mathbb{E}(z(t))$ since the second integral is explicit for this $\sigma$.

Remark 6.9. Since for positive constant communication rate the deterministic Cucker-Smale is always flocking, the introduction of noises in the previous section only introduced in some cases new $\left(\mathbb{L}^{2}\right)$ non-flocking properties.

But here, for linear $\sigma$ we obtain, whatever $\psi$ and the initial condition are, almost sure flocking, so that this time the noise can help to (almost surely) flock, since for the classical communication rate (1.2), we only know that flocking holds true for some initial conditions in the deterministic case $(D=0)$ when $r \geq \frac{1}{2}$.

Comparing swarming and flocking is also easy. Indeed, when (3.8) is satisfied, if the process is $\mathbb{L}^{2,2}$ swarming, the local martingale term of $\left\langle x_{i j}, v_{i j}\right\rangle$, given by

$$
\int_{0}^{t}\left(x_{i}^{*}(s)-x_{j}^{*}(s)\right)\left(\sigma\left(v_{i}(s)\right)-\sigma\left(v_{j}(s)\right)\right) d w(s)
$$

is a true $\mathbb{L}^{2}$ martingale once $\sigma$ is globally Lipschitz (recall that swarming means boundedness for both the expectations of $\left|v_{i}-v_{j}\right|^{2}$ and $\left|x_{i}-x_{j}\right|^{2}$ ). In addition, it is easily seen that, if $\psi$ is bounded, condition (2) in Lemma 3.11 is satisfied under the $\mathbb{L}^{2,2}$ swarming assumption (recall that this assumption includes $\left.\sup _{t} \mathbb{E}\left(\left|v_{i}(t)-v_{j}(t)\right|^{2}\right)<+\infty\right)$. Hence

Proposition 6.10. In the situation of theorem 6.8, assume that (3.8) is satisfied and that $\psi$ is bounded. Then $\mathbb{L}^{2,2}$ swarming implies $\mathbb{L}^{2,2}$ flocking. 
Of course (1) in theorem 6.8 is not fully satisfactory, since it is reasonable to consider models where the communication rate decays with the distance between particles as in (1.2). Let us consider such cases assuming that (3.8) is in force. Define

$$
\psi_{l}(r)=\min _{0 \leq u \leq r} \psi(u),
$$

and

$$
T_{r}=\inf \left\{s \geq 0 ; \max _{i, j}\left|x_{i}(s)-x_{j}(s)\right| \geq r\right\}
$$

\section{Remark 6.13. Back to the deterministic model.}

Assume that $\sigma=0$, hence consider the deterministic model. First of all $d z(t) \leq 0$, so that $z(t) \leq z(0)$ i.e. for all $(i, j), \sup _{t}\left|v_{i}(t)-v_{j}(t)\right|<+\infty$. Hence, according to Proposition 6.10 (where one can forget all the expectations and squares), the process is flocking as soon as $\sup _{t}\left|x_{i}(t)-x_{j}(t)\right|<+\infty$ for all $(i, j)$. But for $t \leq T_{r}$,

$$
d z(t) \leq-2 \lambda \psi_{l}\left(r^{2}\right) z(t)
$$

so that $z(t) \leq z(0) e^{-2 \lambda \psi_{l}\left(r^{2}\right) t}$ and

$$
\begin{aligned}
\left|x_{i}(t)-x_{j}(t)\right| & \leq\left|x_{i}(0)-x_{j}(0)\right|+\int_{0}^{t}\left|v_{i}(s)-v_{j}(s)\right| d s \\
& \leq\left|x_{i}(0)-x_{j}(0)\right|+\int_{0}^{t} z^{\frac{1}{2}}(s) d s \\
& \leq\left|x_{i}(0)-x_{j}(0)\right|+\frac{z^{\frac{1}{2}}(0)}{\lambda \psi_{l}\left(r^{2}\right)}\left(1-e^{-\lambda \psi_{l}\left(r^{2}\right) t}\right),
\end{aligned}
$$

i.e. for all $(i, j)$,

$$
\sup _{t \leq T_{r}}\left|x_{i}(t)-x_{j}(t)\right| \leq\left|x_{i}(0)-x_{j}(0)\right|+\frac{z^{\frac{1}{2}}(0)}{\lambda \psi_{l}\left(r^{2}\right)} .
$$

In particular if for all $(i, j),\left|x_{i}(0)-x_{j}(0)\right|+\frac{z^{\frac{1}{2}}(0)}{\lambda \psi_{l}\left(r^{2}\right)}<r$ then $T_{r}=+\infty$ and the system is flocking.

Choosing $r_{0}=\max _{i, j}\left|x_{i}(0)-x_{j}(0)\right|$ and some $C>1$, it is thus enough that

$$
z^{\frac{1}{2}}(0) \leq \lambda r_{0}(C-1) \psi_{l}\left(C^{2} r_{0}^{2}\right) \text {. }
$$

We recover that if the decay to 0 of $\psi_{l}(r)$ is (strictly) slower than $r^{-\frac{1}{2}}$, the system is flocking for all initial conditions (we may let $C$ go to infinity), while if it is faster, one has to choose the initial conditions in such a way that (6.15) (where one can optimize in $C$ ) is satisfied. Note that we are far from the optimal conditions, but the previous approach is completely elementary.

In the stochastic case, for $t<T_{r}$ (which is now a random stopping time), we have (a.s.)

$$
\ln (z(t))-\ln (z(0)) \leq-\left(2 \lambda \psi_{l}\left(r^{2}\right)-4 K^{2} d^{2}\right) t+\ln (N(t))
$$

where

$$
N_{t}=e^{M(t)-\frac{1}{2}\langle M\rangle(t)}
$$


and $M$. is a martingale whose bracket satisfies $\langle M\rangle(t)=\int_{0}^{t} \alpha(s) d s$ with $|\alpha(t)| \leq 4 K^{2}$. Remark that the remaining stochastic term is the logarithm of an exponential (true) martingale. Of course, if

$$
\theta(r, K)=2 \lambda \psi_{l}\left(r^{2}\right)-4 K^{2} d^{2}>0,
$$

(6.16) shows that $z(t) \rightarrow 0$ as $t \rightarrow+\infty$ almost surely on the set $\left\{T_{r}=+\infty\right\}$. To understand the behavior of $T_{r}$, write

$$
\left|x_{i}\left(t \wedge T_{r}\right)-x_{j}\left(t \wedge T_{r}\right)\right| \leq\left|x_{i}(0)-x_{j}(0)\right|+z^{\frac{1}{2}}(0) \int_{0}^{t} e^{-\left(\lambda \psi_{l}\left(r^{2}\right)-2 K^{2} d^{2}\right) s} N^{\frac{1}{2}}(s) \mathbf{1}_{s<T_{r}} d s .
$$

What we have to do is to control the almost sure behavior of $N(t)$. To this end we first prove a lemma

Lemma 6.18. Let $M(t)$ be a martingale satisfying $\langle M\rangle(t) \leq C t$. Define

$$
S(a, b)=\inf \{t \geq 0, M(t)-b\langle M\rangle(t) \geq a\} .
$$

Then

$$
\mathbb{P}(S(a, b)<+\infty) \leq e^{-2 a b}
$$

Proof. We know that under our assumptions, for all $\eta>0, e^{\eta M(t)-\frac{\eta^{2}}{2}\langle M\rangle(t)}$ is a martingale. Hence

$$
\mathbb{E}\left(e^{\eta M(t \wedge S(a, b))-\frac{\eta^{2}}{2}\langle M\rangle(t \wedge S(a, b))}\right)=1
$$

Choose $\eta=2 b$. This yields

$$
\mathbb{E}\left(\mathbf{1}_{S(a, b)<+\infty} e^{2 b M(t \wedge S(a, b))-2 b^{2}\langle M\rangle(t \wedge S(a, b))}\right) \leq 1 .
$$

Using Lebesgue bounded convergence theorem we may let $t$ go to infinity and obtain the desired result.

Remark 6.19. If $M$ is a standard Brownian motion, it is known that the inequality is an equality.

We deduce from this lemma, that with probability larger than $1-e^{-2 a b}$,

$$
N(t) \leq e^{a+\left(b-\frac{1}{2}\right)\langle M\rangle(t)} \leq e^{a+4\left(b-\frac{1}{2}\right) K^{2} t},
$$

so that

$$
\left|x_{i}\left(s \wedge T_{r}\right)-x_{j}\left(s \wedge T_{r}\right)\right| \leq\left|x_{i}(0)-x_{j}(0)\right|+\frac{z^{\frac{1}{2}}(0) e^{\frac{a}{2}}}{\lambda \psi_{l}\left(r^{2}\right)-2 K^{2} d^{2}-2 K^{2}\left(b-\frac{1}{2}\right)},
$$

provided $\lambda \psi_{l}\left(r^{2}\right)>2 K^{2} d^{2}+2 K^{2}\left(b-\frac{1}{2}\right)$.

Thus, on $\{S(a, b)=+\infty\}$ we may let $s$ go to infinity and get that on $\left\{T_{r}<+\infty\right\}$,

$$
r \leq\left|x_{i}(0)-x_{j}(0)\right|+\frac{z^{\frac{1}{2}}(0) e^{\frac{a}{2}}}{\lambda \psi_{l}\left(r^{2}\right)-2 K^{2} d^{2}-2 K^{2}\left(b-\frac{1}{2}\right)},
$$

which is no more random. Hence, if (6.20) is not satisfied, we have

$$
\mathbb{P}\left(T_{r}=+\infty, S(a, b)=+\infty\right) \geq 1-e^{-2 a b} .
$$

We have thus obtained: 
Theorem 6.21. In the situation of theorem 6.8 assume in addition that (3.8) is in force. Let $r>0$. Let $a, b>0$. Assume that

- $\quad \lambda \psi_{l}\left(r^{2}\right)>2 K^{2}\left(d^{2}+\left(b-\frac{1}{2}\right)\right)$ (replace $d^{2}$ by 1 when $\sigma$ is diagonal, and by 1 if the diagonal term only depends on the corresponding coordinate) where $\psi_{l}$ is defined in (6.11),

- $\quad$ the initial condition satisfies, for all $(i, j)$,

$$
\left|x_{i}(0)-x_{j}(0)\right|+\frac{z^{\frac{1}{2}}(0) e^{\frac{a}{2}}}{\lambda \psi_{l}\left(r^{2}\right)-2 K^{2}\left(d^{2}+\left(b-\frac{1}{2}\right)\right)}<r
$$

where $z(0)=\sum_{k=1}^{N}\left|v_{k}(0)-\bar{v}(0)\right|^{2}$.

Then the system (6.2) is flocking with a probability larger than $1-e^{-2 a b}$.

The previous result is apparently the first one dealing with "conditional flocking" (i.e. flocking for a subset of initial conditions) in a stochastic context (the results in [CM08] have some similarities but are actually different since they deal with approximate flocking before some stopping time).

Remark 6.22. Remark that when $K=0$ corresponding to a constant $\sigma$, we may take any $b$ going to infinity and $a$ going to 0 so that $b a$ goes to infinity. We thus obtain almost sure flocking under the same initial conditions than for the deterministic result (in particular for any initial condition if $r \psi_{l}\left(r^{2}\right) \rightarrow+\infty$ as $\left.r \rightarrow+\infty\right)$. This is not surprising since the microscopic variables satisfy the deterministic system of differential equations. Only the center of mass is driven by some Brownian motion.

Also notice that when $\psi_{l}$ is bounded from below, we recover the almost sure statement in Theorem 6.8 , taking $b=\frac{1}{2}, r=+\infty$ and finally letting $a$ go to infinity.

Finally remark that on $\mathbb{T}_{r}=+\infty, \psi_{l}$ is bounded from below by $\psi_{l}\left(r^{2}\right)$, so that according to (6.16) and the law of large numbers for the martingale $N_{t}, z(t)$ goes to 0 at an exponential (random) rate (depending on $\sup _{t}\left(N_{t} / t\right)$ ), or if one prefers, for any $\kappa<\lambda \psi_{l}\left(r^{2}\right)-2 K^{2} d^{2}$, there exists a random time $\tau_{\kappa}$ such that for $t>\tau_{\kappa}$ the decay of $z(t)$ to 0 is at least $C e^{-\kappa\left(t-\tau_{\kappa}\right)}$. $\tau_{\kappa}$ is simply the last time $N_{t} / t$ is bigger than $\lambda \psi_{l}\left(r^{2}\right)-2 K^{2} d^{2}-\kappa$.

\subsection{Study of (6.1).}

Let us turn to (6.1). Looking at the calculations (5.3) we see that we can mimic what we have just done with the following main modifications: replace $4 K^{2} d^{2}$ by $\frac{2(N+1)}{N^{2}} \max _{i, j}\left\|\sigma_{i, j}^{2}\right\|_{\infty}$ and for the variance of the martingale part $4 K^{2}$ by $4 \max _{i, j}\left\|\sigma_{i, j}^{2}\right\|_{\infty}$. In the very particular case where for all $(i, j), \sigma_{i j}=\sigma$ for some constant $\sigma$, we can argue as in Theorem 5.5 (3).

Hence we only state a general result whose proof is left to the reader :

Theorem 6.23. Consider (6.1). Assume that the processes $\psi_{i j}(t)=\psi_{i j}(v(t), x(t))$ where all the functions $\psi_{i j}$ are local Lipschitz, non-negative and satisfy $\psi_{i j}=\psi_{j i}$, that the processes $\sigma_{i, j}(t)=\sigma_{i, j}(x(t), v(t))$ where all the functions $\sigma_{i j}$ are local Lipschitz, bounded and satisfy $\sigma_{i j}=\sigma_{j i}$. Define $\psi_{\min }$ and $\psi_{\max }$ as in (6.7). Then:

(1) for all initial state $(v(0), x(0)) \in \mathbb{L}^{2}$ the system admits a unique non-explosive (global) strong solution. 
(3) If $\lambda \psi_{\max }<\frac{N+1}{N^{2}} \min _{i, j} \inf _{t \geq 0} \sigma_{i, j}^{2}(t)$ then the system is not $\mathbb{L}^{2}$ flocking.

(4) If $\lambda \psi_{\min }>\frac{N+1}{N^{2}} \max _{i, j}\left\|\sigma_{i, j}^{2}\right\|_{\infty}-\frac{2}{N} \min _{i, j}\left\|\sigma_{i, j}^{2}\right\|_{\infty}$ the system is almost surely flocking.

(5) If $\sigma_{i j}=\sigma$ for all pair $(i, j)$ and some constant $\sigma$, the system is always almost surely flocking, whatever $\psi$ is.

Assume in addition that $\psi$ satisfies (3.8). Then

(6) if $\psi$ is bounded, $\mathbb{L}^{2,2}$ swarming implies $\mathbb{L}^{2,2}$ flocking.

(7) Let $r>0, a, b>0$. Assume that

(a)

$$
\lambda \psi_{l}\left(r^{2}\right)>\left(2 b+\frac{N+1}{N^{2}}\right) \max _{i, j}\left\|\sigma_{i, j}^{2}\right\|_{\infty}-\frac{2}{N} \min _{i, j} \inf _{t \geq 0} \sigma_{i, j}^{2}(t)
$$

where $\psi_{l}$ is defined in (6.11),

(b) the initial condition satisfies, for all $(i, j)$,

$$
\begin{aligned}
& \left|x_{i}(0)-x_{j}(0)\right|+\frac{z^{\frac{1}{2}}(0) e^{\frac{a}{2}}}{\lambda \psi_{l}\left(r^{2}\right)-\left(2 b+\frac{N+1}{N^{2}}\right) \max _{i, j}\left\|\sigma_{i, j}^{2}\right\|_{\infty}+\frac{2}{N} \min _{i, j} \inf _{t \geq 0} \sigma_{i, j}^{2}(t)}<r \\
& \text { where } z(0)=\sum_{k=1}^{N}\left|v_{k}(0)-\bar{v}(0)\right|^{2} .
\end{aligned}
$$

Then the system is flocking with a probability larger than $1-e^{-2 a b}$.

Once again when $\sigma$ goes uniformly to 0 we recover the deterministic situation just by choosing $a$ and $b$ in an appropriate way.

\subsection{A simple example with $N=2$ for (6.2).}

The reader certainly remarked that, when $\sigma$ is constant in (6.2), changing $v(t)$ into $v(t)-$ $\sigma w(t)$, the system obeys the deterministic dynamics (this is the favorite random situation for the non probabilists). Hence in this situation, conditional flocking or non flocking holds with probability 1 , depending on the deterministic behavior.

It should be interesting to exhibit an example (even with two particles) where almost sure flocking holds with a strictly positive probability strictly less than 1 . This seems to be a hard task. However we shall study in details simple examples to better understand what happens. For reasons we shall explain later, we shall consider the case $N=2$ and $d=1$.

\subsubsection{An explicit deterministic example.}

Take $N=2, d=1$ and look at the deterministic system

$$
\begin{aligned}
& d v_{1}(t)=-2 \frac{v_{1}(t)-v_{2}(t)}{1+\left|x_{1}(t)-x_{2}(t)\right|^{2}} d t \\
& d v_{2}(t)=-2 \frac{v_{2}(t)-v_{1}(t)}{1+\left|x_{1}(t)-x_{2}(t)\right|^{2}} d t
\end{aligned}
$$


with an initial condition $v_{1}(0)=-v_{2}(0), x_{1}(0)=-x_{2}(0)$. The unique solution satisfies $v_{1}(t)=-v_{2}(t), x_{1}(t)=-x_{2}(t)$ and the difference $v(t)=v_{1}(t)-v_{2}(t)=2 v_{1}(t)$ satisfies

$$
d v(t)=-\frac{v(t)}{1+|x(t)|^{2}} d t
$$

so that

$$
v(t)-v(0)=\arctan (x(0))-\arctan (x(t))
$$

and

$$
x(t)-x(0)=\int_{0}^{t}(v(0)+\arctan (x(0))-\arctan (x(s))) d s .
$$

We confess that we do not know how to solve the O.D.E.

$$
x^{\prime}(t)=c-\arctan (x(t)) .
$$

Nevertheless we can study the qualitative behavior of the system. Indeed one can notice the following points

(1) if $v(0)=0$ the unique solution is $v(t)=0$ and $x(t)=x(0)$.

(2) It follows that if $v(0) \geq 0$, then the solution $v(t) \geq 0$ for all $t \geq 0$. Indeed if $v($. reaches 0 then it is sticked at 0 according to the previous point.

If one prefers, one can also write

$$
v(t)=v(0) e^{-\int_{0}^{t} \frac{d s}{1+|x(s)|^{2}}} \geq v(0) e^{-t} .
$$

Hence $x($.$) is non decreasing, so that assuming that x(0) \geq 0, \lim _{t \rightarrow+\infty} x(t)=x(\infty) \leq$ $+\infty$.

Now consider a solution such that $x(0)=0$ (for simplicity) and $v(0) \geq 0$. If $x(\infty)<+\infty$, since $x(t) \leq x(\infty),(v)^{\prime}(t) \leq-\frac{v(t)}{1+|x(\infty)|^{2}}$ so that $v(t) \rightarrow 0$ as $t \rightarrow+\infty$ at an exponential rate. Thus, $0=v(0)-\arctan (x(\infty))$ by letting $t$ go to infinity. Similarly if $x(\infty)=+\infty$, $\lim _{t \rightarrow+\infty} v(t)=v(0)-\frac{\pi}{2} \geq 0$ since $v(t) \geq 0$.

Hence

(1) if $x(0)=0$ and $0 \leq v(0)<\frac{\pi}{2}, x(\infty)<+\infty$ so that $v(t) \rightarrow 0$ and $x(t) \rightarrow \tan (v(0))$, the system is flocking,

(2) if $x(0)=0$ and $v(0) \geq \frac{\pi}{2}, x(t) \rightarrow+\infty$ and $v(t) \rightarrow v(0)-\frac{\pi}{2}$, so that the system is not flocking.

\subsubsection{Back to the stochastic model.}

Consider the general case with $\psi$ satisfying (3.8). If we add a stochastic term such that $\sigma(-v)=-\sigma(v)$ (assuming as before that $\sigma$ is $K$-Lipschitz) we still have $v_{1}(t)=-v_{2}(t)$, $x_{1}(t)=-x_{2}(t)$ and the difference $v(t)$ satisfies

$$
d v(t)=-\psi\left(|x(t)|^{2}\right) v(t) d t+2 \sigma\left(\frac{v(t)}{2}\right) d w(t) .
$$

Again the unique solution starting from $v(0)=0$ and $x(0)$ is $v(t)=0, x(t)=x(0)$, so that using the Markov property, if $v(0) \geq 0, v(t) \geq 0$ for all $t \geq 0$. For simplicity again we assume 
that $x(0)=0$ and $v(0)>0$.

Hence, up to the first time $v($.$) reaches 0$ (and then is sticked at 0 ) we may write

$$
d(\ln (v(t)))=-\psi\left(|x(t)|^{2}\right) d t-2 \frac{\sigma^{2}(v(t) / 2)}{v^{2}(t)} d t+2 \frac{\sigma(v(t) / 2)}{v(t)} d w(t) .
$$

Here again we have

$$
v(t)=v(0) e^{-\int_{0}^{t} \psi\left(|x(s)|^{2}\right) d s} e^{N(t)-\frac{1}{2}\langle N\rangle(t)}
$$

where $N($.$) is a \mathbb{L}^{2}$ martingale, so that $v($.$) does not hit 0$ in finite time a.s. But this representation allows us to obtain more information. Indeed lemma 6.18 tells us that for any $a>0$,

Hence

$$
\mathbb{P}\left(\sup _{t \geq 0}\left(N(t)-\frac{1}{2}\langle N\rangle(t)\right) \geq a\right) \leq e^{-a}
$$

$$
\mathbb{P}\left(\limsup _{t \rightarrow+\infty} v(t)=+\infty\right) \leq \mathbb{P}\left(\limsup _{t \rightarrow+\infty}\left(N(t)-\frac{1}{2}\langle N\rangle(t)\right)=+\infty\right)=0 .
$$

We know that the martingale term in (6.24) satisfies almost surely,

$$
\lim _{t \rightarrow+\infty} \frac{1}{t} \int_{0}^{t} \frac{\sigma(v(s) / 2)}{v(s)} d w(s)=0 .
$$

Assume that

$\sigma$ is of class $C^{1}$ with a bounded derivative, $\sigma^{\prime}(0)>0$, and $\sigma(v)>0$ for all $v>0$.

As a consequence

$$
\inf _{0 \leq v \leq a} \frac{\sigma(v)}{v}=\sigma_{\min }(a)>0 .
$$

Notice that (6.27) is satisfied in particular if $\sigma(v) \geq C v$ for some $C>0$ and all $v \geq 0$, which is nothing else than a simple extension of the linear case, since in this case, for $v \geq 0$, $C v \leq \sigma(v) \leq K v$.

Now for almost all given $\omega, \lim \sup v(t)(\omega)=v_{\max }(\omega)<+\infty$, so that

$$
\frac{1}{t} \int_{0}^{t} \frac{\sigma^{2}(v(s)(\omega) / 2)}{v^{2}(s)(\omega)} d s \geq \frac{1}{4} \sigma_{\min }^{2}\left(v_{\max }(\omega) / 2\right) .
$$

It follows that $\ln (v(t)) \rightarrow-\infty$ i.e. $v(t) \rightarrow 0$, and that the latter convergence is exponential (at least $e^{-\frac{1}{2} \sigma_{\min }^{2}\left(v_{\max }^{2}(\omega) / 2\right) t}$ ), so that $x(t)$ is almost surely bounded, and the system is almost surely flocking. We have proved

Proposition 6.29. Consider (6.2) for $N=2, d=1$ with $\sigma(v)=-\sigma(-v)$, and assume that (3.8) and (6.27) are satisfied. Then the system is always almost surely flocking.

Remark 6.30. (1) In the previous proof, since we know that $v(t)$ goes to 0 , using L'Hospital's and Cesaro's rules, we obtain

$$
\lim _{t \rightarrow+\infty} \frac{1}{t} \int_{0}^{t} 2 \frac{\sigma^{2}(v(s) / 2)}{v^{2}(s)} d s=\frac{1}{2}\left(\sigma^{\prime}(0)\right)^{2},
$$

which is no more random. But one has to be careful because this limit is not uniform in $\omega$. 
(2) Of course what we have just done is to show (exponential) stability for some stochastic differential equation. Indeed, since we are in dimension 1 and the interaction term is non-positive, we know that $v(t) \leq u(t)$ where $u($.$) solves$

$$
d u(t)=\sigma(u(t)) d w(t) .
$$

Our proof shows that $u(t) \rightarrow 0$ at an exponential rate almost surely.

(3) Assume that $\sigma$ is compactly supported, say by $[-M, M]$. Thus, (6.27) is not fulfilled. Take $\psi(u)=\frac{1}{1+u^{2}}$. If $v(0)>M+\frac{\pi}{2}$, then $v($.$) behaves like the deterministic$ model (hence stays larger than $M$ ) and does not flock. Hence in (6.27) the behavior of $\sigma(v) / v$ near the origin is not sufficient to control flocking.

(4) However if we only skip the assumption $\sigma^{\prime}(0)>0$ in (6.27) and replace it by $\sigma^{\prime}(0)=0$, the previous proof shows that $\liminf _{t \rightarrow+\infty} v(t)=0$. Indeed if not we get again a lower bound as in (6.28), by taking the minimum of $\sigma(v / 2) / v$ on the interval $\left[v_{\min }=\lim \inf v(t), v_{\max }=\lim \sup v(t)\right]$.

Is it possible to get flocking while the process $u($.$) in (2) does not flock, that is to get an$ example where the interaction $\psi$ really does matter ? Here is almost one.

Choose

Then if $0 \leq v \leq v_{\max }$,

$$
\sigma(v / 2)=\frac{v^{\frac{3}{2}}}{1+v^{2}}
$$

$$
\frac{v}{\left(1+v_{\max }^{2}\right)^{2}} \leq \frac{\sigma^{2}\left(\frac{v}{2}\right)}{v^{2}} \leq v
$$

Hence, since $v_{\max }=\limsup v(t)<+\infty$ almost surely, if $\int_{0}^{+\infty} \frac{\sigma^{2}(v(s) / 2)}{v^{2}(s)} d s<+\infty$ (resp. $=+\infty), \sup _{t} x(t) \leq \int_{0}^{+\infty} v(s) d s<+\infty($ resp. $=+\infty)$ almost surely, so that in all cases

$$
\int_{0}^{+\infty}\left(\psi\left(|x(t)|^{2}\right)+2 \frac{\sigma^{2}(v(t) / 2)}{v^{2}(t)}\right) d t=+\infty .
$$

Come back to the expression (6.25). We know that $e^{N(t)-\frac{1}{2}\langle N\rangle(t)}$ is almost surely finite, so that if $\int_{0}^{+\infty} \psi\left(|x(t)|^{2}\right) d t=+\infty, v(t) \rightarrow 0$. In addition, lemma 6.18 tells us that for any $a>0$,

$$
\mathbb{P}\left(\sup _{t \geq 0}(N(t)-b\langle N\rangle(t)) \geq a\right) \leq e^{-2 a b},
$$

so that for $b<\frac{1}{2}, e^{N(t)-b\langle N\rangle(t)}$ is almost surely finite. Thus if

$$
\langle N\rangle(t)=4 \int_{0}^{t} \frac{\sigma^{2}(v(s) / 2)}{v^{2}(s)} d s \rightarrow+\infty,
$$

$e^{N(t)-\frac{1}{2}\langle N\rangle(t)}$ goes to 0 and so does $v(t)$ again.

But we do not know whether $\sup _{t} x(t)$ is always a.s. finite or not, so that we do not know whether the process is flocking or not.

Finally, in the particular case $\psi(u)=\frac{1}{1+u^{2}}$, if $v(0)>\frac{\pi}{2}, x(0)=0$, the system is not $\mathbb{L}^{1}$ flocking. Indeed, taking the expectation $(v(t) \geq 0)$ we have

$$
\mathbb{E}(v(t))=v(0)-\mathbb{E}(\arctan (x(t))) \geq v(0)-\frac{\pi}{2}>0 .
$$


So once again, $\mathbb{L}^{p}$ flocking is much more demanding.

\section{Comments and simulations.}

What kind of (temporary) conclusions can we draw after this study ?

(1) All the models we have discussed in the introduction (except (1.9) for which we do not have a convincing interpretation) have their "reasonable" physical (or biological) interpretation and at the same time suffer potential criticism. They are only models and certainly not a description of reality.

(2) Too independent noises destroy the collective behavior (without any politically correct reference).

(3) Random environment depending in a certain way of the positions can also destroy the collective behavior.

(4) Noises whose variances depend either linearly on the velocities or on the differences between velocities may help, at least at the almost sure level, to flock. But actually in many of these situations, the communication between individuals is simply a perturbation of a stochastic system which is already stable (though, except in a very few number of particular cases, one cannot reduce the study to the use of the theory of stability of S.D.E. as detailed in the book [Mao07]).

(5) Due to the previous item, $\mathbb{L}^{2}$ flocking is presumably more convincing.

We shall now illustrate our results (and the situations that are not covered by our results) with some simulations. First we shall consider the system (6.2)

$$
d v_{i}(t)=-\frac{\lambda}{N} \sum_{j=1}^{N} \psi_{i j}(t)\left(v_{i}(t)-v_{j}(t)\right) d t+\sigma\left(v_{i}(t)\right) d w(t) .
$$

In all the section we will choose

$$
\psi_{i j}(t)=\psi\left(\left|x_{i}(t)-x_{j}(t)\right|\right) \quad \text { with } \quad \psi(u)=\left(1+u^{2}\right)^{-1}
$$

in dimension $d=2$ with $N=9$ particles and communication intensity $\lambda=10$.

We shall consider two basic set of initial configurations $\left(x^{1}(0), v^{1}(0)\right)$ and $\left(x^{2}(0), v^{2}(0)\right)$ given by $x^{1}(0)=0$

$$
\begin{aligned}
v^{1}(0) & =\left(\begin{array}{ccccccccc}
-0.4 & 0.2 & -0.3 & -0.3 & -0.1 & -0.2 & 0.2 & 0.5 & 0.2 \\
0.4 & -0.1 & 0.2 & 0.5 & 0.3 & 0.1 & -0.3 & 0.2 & 0.3
\end{array}\right) \\
x^{2}(0) & =\left(\begin{array}{ccccccccc}
1 & 0 & 0 & 0 & 0 & 0 & 0 & 0 & 0 \\
-4 & 0 & 0 & 0 & 0 & 0 & 0 & 0 & 0
\end{array}\right) \\
v^{2}(0) & =\left(\begin{array}{cccccccccc}
-0.3 & 2 & -0.5 & -1.5 & -0.1 & -0.2 & 1.2 & 0.5 & 1.5 \\
0.7 & -0.6 & 2.1 & 0.4 & 0.8 & 2.6 & -3.4 & -0.6 & 0.2
\end{array}\right)
\end{aligned}
$$

Define $z(0)=\sum_{k=1}^{N}\left|v_{k}(0)-\bar{v}(0)\right|^{2}$ and $M_{x}(0)=\max _{i, j}\left|x_{i}(0)-x_{j}(0)\right|$. Recall the discussion preceding (6.15) ensuring flocking starting from $(x(0), v(0))$, i.e. we want to find some $r>0$ such that the function $g$ defined by

$$
g(r)=M_{x}(0)+\frac{\sqrt{z(0)}}{\lambda}\left(1+r^{2}\right)-r
$$


is negative at $r$. This is equivalent to the following

$$
\sqrt{z(0)}<\frac{\lambda}{2}\left(\sqrt{M_{x}(0)^{2}+\frac{1}{4}}-M_{x}(0)\right)
$$

and it is easy to show that the first set of initial data satisfies this condition, while the second one does not (see Figure 1 below). In the sequel we shall use modified initial data of the form $\left(x^{i}(0), \theta v^{i}(0)\right)$ for some given $\theta$ 's and will plot the function $g$ to see wether the corresponding initial data do satisfy the condition or not.

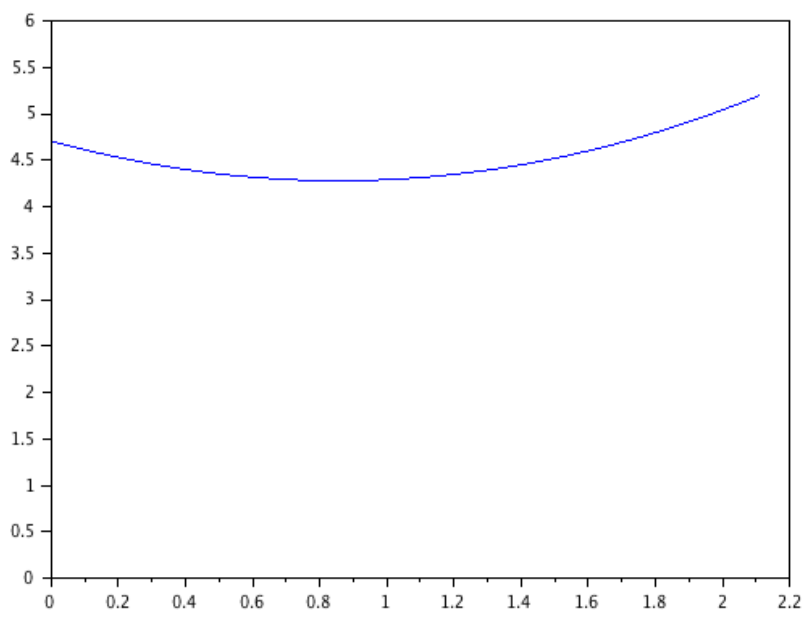

Figure 1. $g(r)$ in case $\left(x^{2}(0), v^{2}(0)\right)$

We shall now plot several simulations of the stochastic model or numerical approximations in the deterministic case. In both cases the numerical scheme is a simple explicit Euler scheme. On each Figure we draw the evolution in time of

$$
t \mapsto\left(\sum_{i=1}^{N}\left|v_{i}(t)-\bar{v}(t)\right|^{2}\right)^{\frac{1}{2}}
$$

for both the stochastic and the deterministic system. Recall that we do not have theoretical result for the flocking property for the deterministic system once condition (6.15) is not satisfied.

In the next Figure 2 we choose $\sigma(v)=v$ and initial condition $\left(x^{2}(0), v^{2}(0)\right)$. According to Theorem 6.8 (2), we know that the stochastic system is almost surely flocking, but we do not know about $\mathbb{L}^{2}$ flocking.

We observe that in this case the deterministic system flocks too and a reasonably quick convergence for the stochastic system.

Next still with $\sigma(v)=v$ we change the initial configuration by choosing $\left(x^{2}(0), 5 v^{2}(0)\right)$. In this situation we see that the deterministic system does not flock anymore, while the 


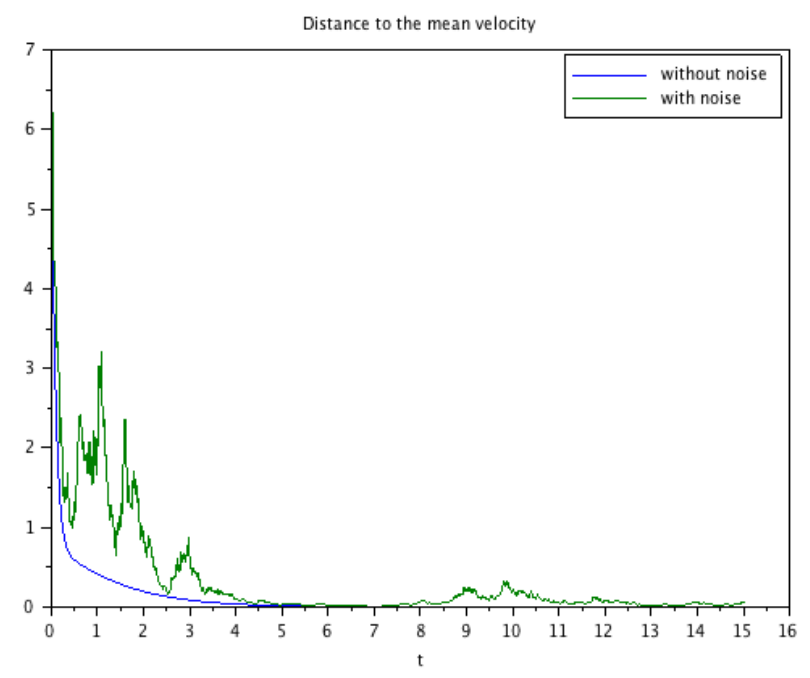

Figure 2. $t \mapsto\left(\sum_{i=1}^{N}\left|v_{i}(t)-\bar{v}(t)\right|^{2}\right)^{\frac{1}{2}}$ for $\sigma(v)=v$ in case $\left(x^{2}(0), v^{2}(0)\right)$

stochastic system almost surely flocks. In Figure 3 we plot the evolution of the velocities on the right hand side, but also, on the left hand side, the evolution of $t \mapsto \max _{i, j}\left|x_{i}(t)-x_{j}(t)\right|$.
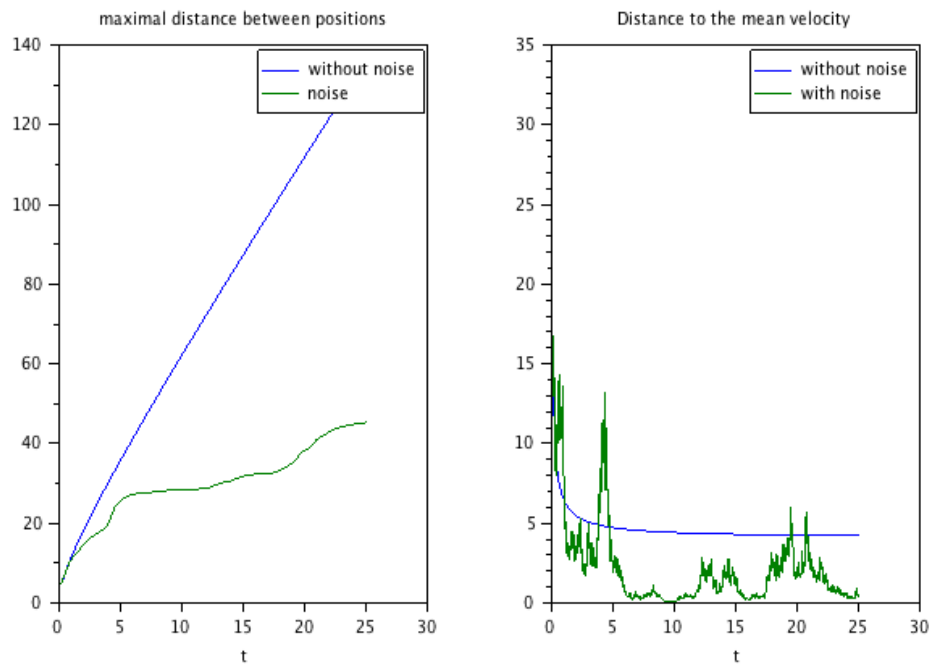

FiguRE 3. $\left(x^{2}(0), 5 v^{2}(0)\right) \quad, \quad \sigma=v$ first case.

The next two figures are obtained with the same data (be careful with the vertical scale which is not the same for each figure). The convergence to 0 in the stochastic case can be surprisingly quick (Figure 5), very slow (Figure 4 where the fluctuations size presumably 
indicates that there is no $\mathbb{L}^{2}$ flocking) or similar to the previous case (Figure 3 where we also observe a chaotic stabilization of the positions).

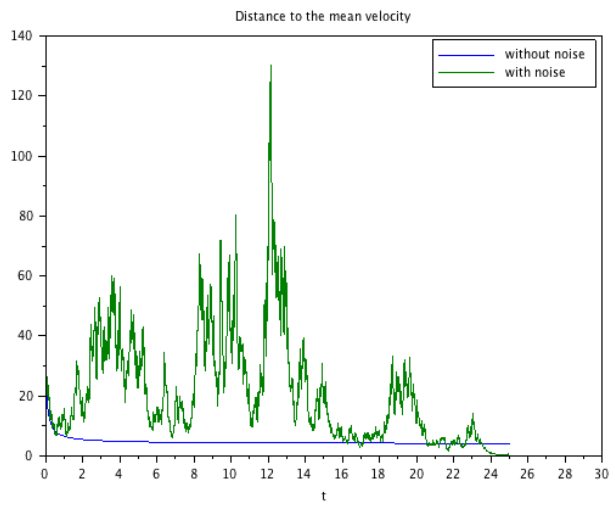

Figure 4. $\left(x^{2}(0), 5 v^{2}(0)\right)$, $\sigma=v$ second case.

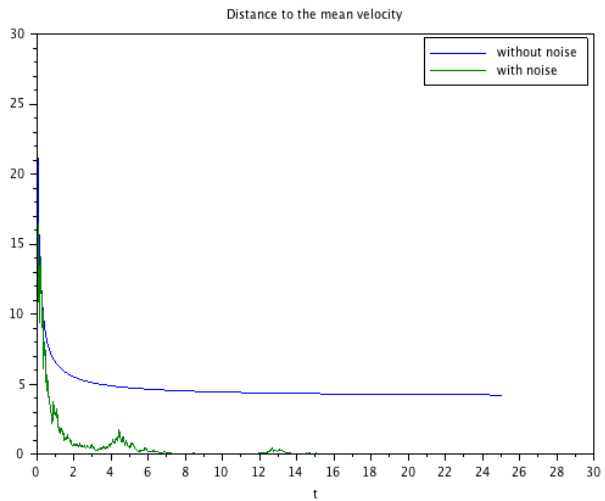

Figure 5. $\left(x^{2}(0), 5 v^{2}(0)\right)$, $\sigma=v$ third case.

The next situation we want to illustrate is the one of Theorem 6.21. To this end we choose $\sigma\left(v^{i}\right)=1+\sin v^{i}$ for each of the two coordinates $v^{i}$ of $v$, hence a diagonal $\sigma$. Since $\sigma$ is 1 -Lipschitz, we choose $b=1 / 2$ and $a=\ln 2$ so that if the initial conditions satisfy the assumption in Theorem 6.21, the latter tells us that the stochastic system flocks with a probability larger than or equal to $\frac{1}{2}$.

To fulfill this assumption we choose this time $\left(x^{1}(0), 0,1 v^{1}(0)\right)$ as initial conditions. We thus know that the deterministic system is flocking. The next figure 6 plot the condition showing that some $r$ can be found, while figure 7 presents one example of simulation. Actually in this case we did not be able to obtain a non-flocking stochastic simulation, showing that, as for sure, our result is far from optimal.

To observe something interesting we have to change the initial condition and thus choose $\left(x^{2}(0), 3 v^{2}(0)\right)$. If we still have the flocking property for the deterministic model, we have observed (as the two examples show) various cases in the stochastic case, with flock or no flock, indicating that flocking may occur with some probability strictly larger than 0 and strictly less than 1 . 


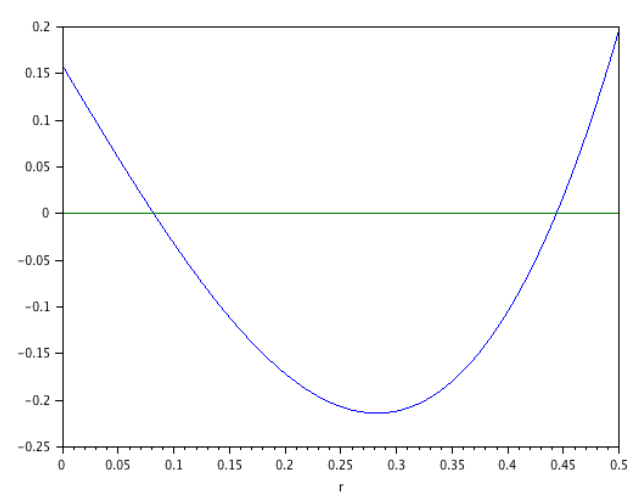

Figure $6 .\left(x^{1}(0), 0.1 v^{1}(0)\right)$ condition

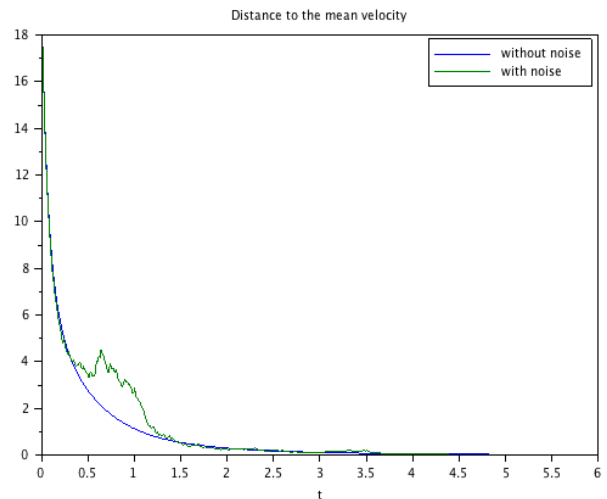

Figure 8. $\left(x^{2}(0), 3 v^{2}(0)\right)$, $\sigma(v)=1+\sin (v)$ with flock

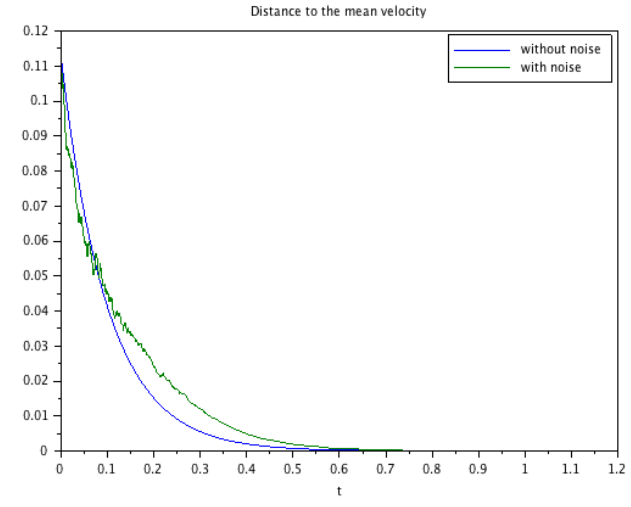

Figure $\quad 7 .\left(x^{1}(0), 0.1 v^{1}(0)\right)$ and $\sigma=1+\sin (v)$.

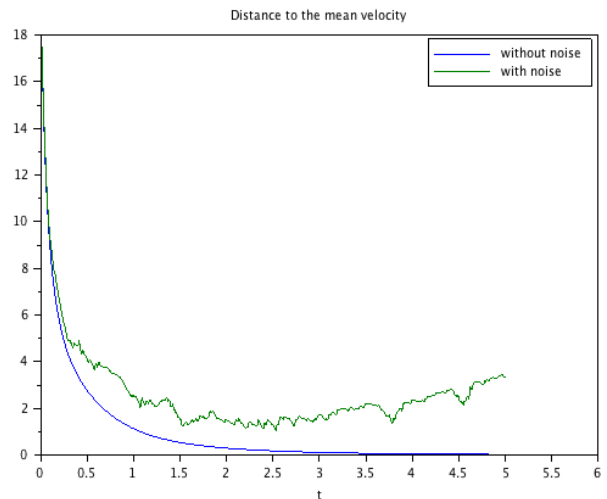

Figure 9. $\left(x^{2}(0), 3 v^{2}(0)\right)$, $\sigma(v)=1+\sin (v)$ with no flock

Finally, we show some simulations when $\sigma$ is a function of $x$ and no more of $v$. As we have seen this situation is completely unclear, even for a constant communication rate. This chaotic behavior is illustrated by the final three pictures where as before we have drawn the behavior of the positions on the left hand side and of the velocities on the right hand side. 

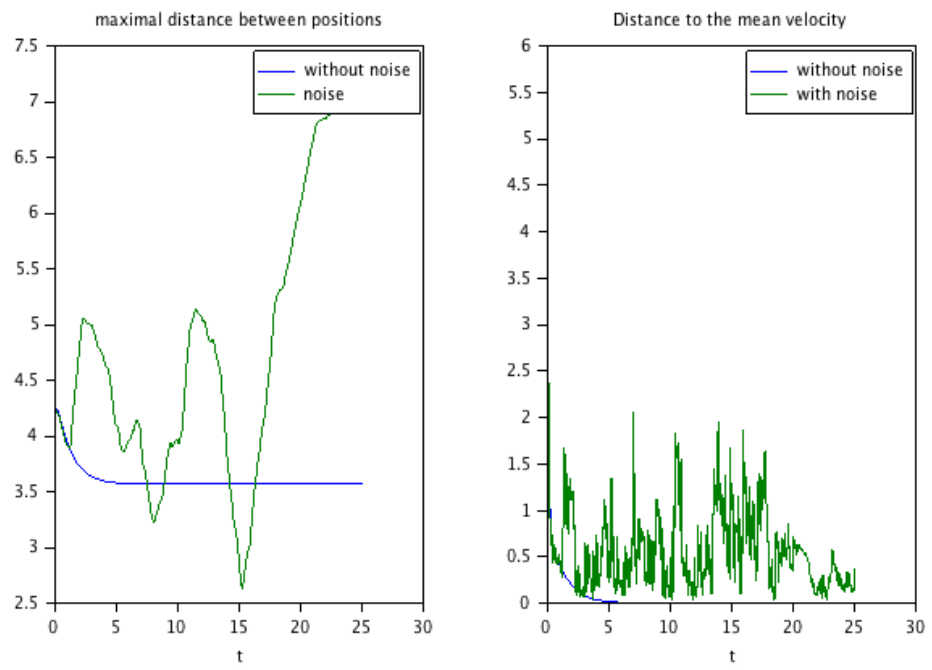

FiguRE 10. $\left(x^{2}(0), v^{2}(0)\right), \sigma=1+\sin (x)$ first case
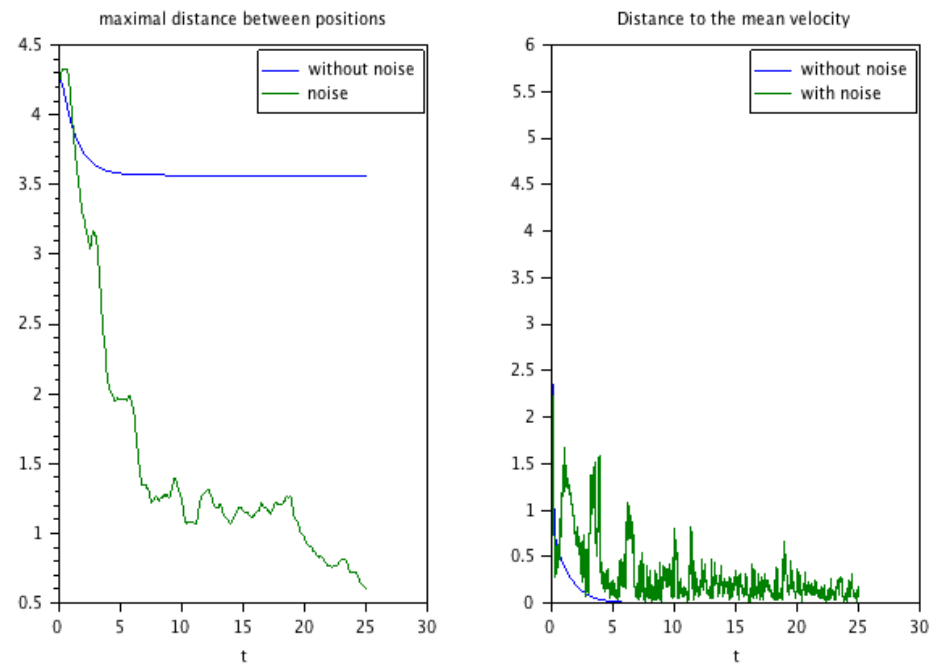

Figure 11. $\left(x^{2}(0), v^{2}(0)\right), \sigma=1+\sin (x)$ with flock

\section{REFERENCES}

[AH10] S. M. Ahn and S-Y Ha. Stochastic flocking dynamics of the Cucker-Smale model with multiplicative white noises. J. Math. Physics, 51:103301, 2010.

[BCnC11] F. Bolley, J. A. Cañizo, and J. A. Carrillo. Stochastic mean-field limit: non-Lipschitz forces and swarming. Math. Models Methods Appl. Sci., 11:2179-2210, 2011.

[CM08] F. Cucker and E. Mordecki. Flocking in noisy environments. J. Math. Pures Appl., 89:278-296, 2008. 

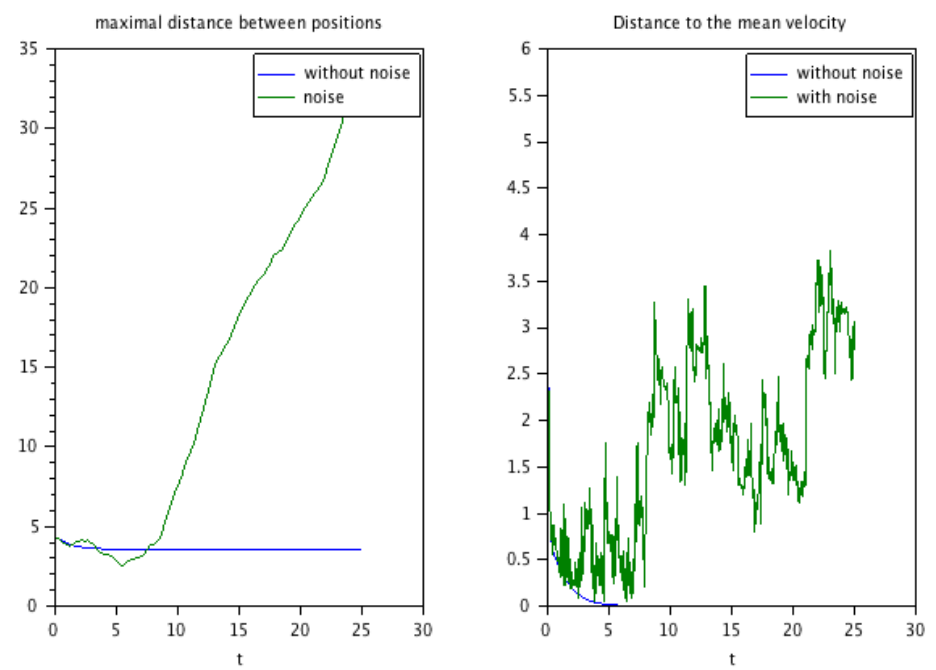

Figure 12. $\left(x^{2}(0), v^{2}(0)\right), \sigma=1+\sin (x)$ with no flock

[CS07a] F. Cucker and S. Smale. Emergent behavior in flocks. IEEE Trans. Automat. Control, 52:852-862, 2007.

[CS07b] F. Cucker and S. Smale. On the mathematics of emergence. Jpn. J. Math., 2:197-227, 2007.

[EHS15] R. Erban, J. Haskovec, and Y. Sun. On Cucker-Smale model with noise and delay. Available on ArXiv 1507.04432 [DS]., 2015.

[HL09] S-Y. Ha and J-G. Liu. A simple proof of the Cucker-Smale flocking dynamics and mean-field limit. Comm. Math. Sci., 7:297-325, 2009.

[HLL09] S-Y. Ha, K. Lee, and D. Levy. Emergence of time-asymptotic flocking in a stochastic Cucker-Smale system. Comm. Math. Sci., 7:453-469, 2009.

[HT08] S-Y. Ha and E. Tadmor. From particle to kinetic and hydrodynamic descriptions of flocking. Kinet. Relat. Models, 1:415-435, 2008.

[Mao07] X. Mao. Stochastic differential equations and applications. Horwood Publishing Limited, 2007.

[MT11] S. Motsch and E. Tadmor. A new model for self-organized dynamics and its flocking behaviour. $J$. Stat. Phys., 144:923-947, 2011.

[Péd16] L. Pédèches. Asymptotic properties of some stochastic Cucker-Smale dynamics. Preprint., 2016.

[SL15] Y. Sun and W. Lin. A positive role of multiplicative noise on the emergence of flocking in a stochastic Cucker-Smale system. Chaos, 25:083118, 2015.

[TLY14] T. V. Ton, N. T. H. Linh, and A. Yagi. Flocking and non flocking in a stochastic Cucker-Smale model. Anal. Appl., 12(1):63-73, 2014.

[VZ12] T. Vicsek and A. Zafeiris. Collective motion. Physics Reports, 517(3):71-140, 2012. 
Patrick CattiauX, Institut de Mathématiques de Toulouse. Université de Toulouse. CNRS UMR 5219., 118 Route de NARBonne, F-31062 Toulouse Cedex 09.

E-mail address: patrick.cattiaux@math.univ-toulouse.fr

Fanny DeleBecQUE,, Institut de Mathématiques de Toulouse. Université de Toulouse. CNRS UMR 5219., 118 Route de Narbonne, F-31062 Toulouse Cedex 09.

E-mail address: fanny.delebecque@math.univ-toulouse.fr

Laure PeDECHeS,, Institut de Mathématiques de Toulouse. Université de Toulouse. CNRS UMR 5219., 118 Route de NARbonne, F-31062 Toulouse CEdex 09.

E-mail address: laure.pedeches@math.univ-toulouse.fr 